\title{
THE RAMSEY PROPERTY FOR OPERATOR SPACES AND NONCOMMUTATIVE CHOQUET SIMPLICES
}

\author{
DANA BARTOŠOVÁ, JORDI LÓPEZ-ABAD, MARTINO LUPINI, AND B. MBOMBO
}

\begin{abstract}
The noncommutative Gurarij space $\mathbb{N} \mathbb{G}$, initially defined by Oikhberg, is a canonical object in the theory of operator spaces. As the Fraïssé limit of the class of finite-dimensional nuclear operator spaces, it can be seen as the noncommutative analogue of the classical Gurarij Banach space. In this paper, we prove that the automorphism group of $\mathbb{N} \mathbb{G}$ is extremely amenable, i.e. any of its actions on compact spaces has a fixed point. The proof relies on the Dual Ramsey Theorem, and a version of the Kechris-Pestov-Todorcevic correspondence in the setting of operator spaces.

Recent work of Davidson and Kennedy, building on previous work of Arveson, Effros, Farenick, Webster, and Winkler, among others, shows that nuclear operator systems can be seen as the noncommutative analogue of Choquet simplices. The analogue of the Poulsen simplex in this context is the matrix state space $\mathbb{N P}$ of the Fraïssé limit $A(\mathbb{N P})$ of the class of finite-dimensional nuclear operator systems. We show that the canonical action of the automorphism group of $\mathbb{N P}$ on the compact set $\mathbb{N P}_{1}$ of unital linear functionals on $A(\mathbb{N P})$ is minimal and it factors onto any minimal action, whence providing a description of the universal minimal flow of Aut $(\mathbb{N P})$.
\end{abstract}

\section{INTRODUCTION}

Dynamics studies, generally speaking, actions of groups on spaces. When the group $G$ under consideration is topological, it is natural to restrict to actions that are continuous. While a classification of arbitrary continuous actions is hopeless, one can hope for a good structure theory after restricting to continuous actions on compact spaces, also called flows or G-flows. In this case, by Zorn's lemma one can conclude that every flow admits a subflow that is furthermore minimal (with respect to inclusion). Thus, in some sense flows that are minimal (i.e. have no nontrivial subflows) can be regarded as building blocks of more general flows. Within the class of minimal flows there is a unique one that is largest or universal, in the sense that it factors onto any minimal flow [15, 26]. Such a universal minimal $G$-flow $M(G)$ is thus a canonical object in the study of the dynamics of $G$, as it encodes fundamental properties of the class of all $G$-flows. For instance, $M(G)$ being reduced to a single point, in which case the group $G$ is called extremely amenable, is equivalent to the assertion

2000 Mathematics Subject Classification. Primary 05D10, 46L07; Secondary 37B05, 46L05.

Key words and phrases. noncommutative Gurarij space, noncommutative Poulsen simplex, extreme amenability, Ramsey property, operator space, operator system, oscillation stability, Dual Ramsey Theorem.

D.B. was supported by the grant FAPESP 2013/14458-9. J.L.-A. was partially supported by the Ministerio de Economía y Competitividad grant MTM2016-76808 (Spain), the Ministerio de Ciencia e Innovación grant PID2019107701GB-I00 (Spain) and the Fapesp grants 2013/24827-1 and 2016/25574-8 (Brazil). M.L. was partially supported by the NSF Grant DMS-1600186 and by the Marsden Fund Fast-Start Grant VUW1816 from the Royal Society of New Zealand. B.M. was supported by Fundação de Amparo à Pesquisa do Estado de São Paulo (FAPESP) postdoctoral grant, processo 12/20084-1. This work was initiated during a visit of J.L.-A. to the Universidade de Sao Pãulo in 2014, and continued during visits of D.B. and J.L.-A. to the Fields Institute in the Fall 2014, a visit of M.L. to the Instituto de Ciencias Matemáticas in the Spring 2015, and a visit of all the authors at the Banff International Research Station in occasion of the Workshop on Homogeneous Structures in the Fall 2015. The hospitality of all these institutions is gratefully acknowledged. 
that every $G$-flow has a fixed point. More generally, obtaining a concrete description of $M(G)$ entails at least in principle a classification of all minimal $G$-flows, which are precisely the factors of $M(G)$. This is especially interesting when $M(G)$ turns out to be "small" or, specifically, metrizable, in which case any minimal $G$-flow is metrizable as well.

While $M(G)$ is never metrizable when $G$ is locally compact and not compact, breakthroughs due to Pestov [49-51] culminating in the work of Kechris, Pestov, and Todorcevic [30] provided several examples of "large" topological groups for which $M(G)$ is metrizable, or even trivial. These groups arise as automorphism groups of mathematical structures satisfying a strong homogeneity property called ultrahomogeneity. Examples of such structures are the linear order of the rationals $(\mathbb{Q},<)$, the Urysohn metric space $\mathbb{U}$, the infinite random graph $\mathcal{R}$, and the countable atomless Boolean algebra $\mathcal{B}$. If $G$ denotes the automorphism group of one of these examples, then $M(G)$ is trivial in the case of the rationals and the Urysohn space, and it is equal to the space of all linear orderings on $\mathcal{R}$ or to the space of natural linear orderings on $\mathcal{B}$, in the case of the infinite Random graphs and the countable atomless Boolean algebra, respectively.

In the case of the automorphism group $G$ of a ultrahomogeneous structure $X$, the Kechris-PestovTodorcevic (KPT) correspondence from [30], later extended by Van The [45], provides a way to compute $M(G)$ by studying the age of $X$, which is the collection of all the "small" (finitely-generated) substructures of $X$. For instance, in the case of the linear order of the rationals, of the Urysohn space, and the infinite random graph, the age is the class of all the finite linear orders, all finite metric spaces, and all finite graphs, respectively. Precisely, the KPT correspondence characterizes extreme amenability of $G$ in terms of a strong combinatorial property of the age of $X$, called the Ramsey property, the name being due to the fact that in the case of finite linear orders it can be seen as a reformulation the finite Ramsey theorem. This provides a combinatorial way to establish extreme amenability of $G$ or to compute $M(G)$ after finding a suitable extremely amenable "large" subgroup. (A different approach, using the method of concentration of measure, was pioneered by Gromov ad Milman [23], who employed it to prove that the group of unitary operators on the Hilbert space endowed with the strong operator topology is extremely amenable.)

Until recently, virtually all examples of application of the KPT correspondence consisted of discrete structures arising in algebra and combinatorics. This has changed in recent years, where the scope of the KPT correspondence has been extended to "continuous" structures from geometry and functional analysis. One can place in this context the main results of [3], where the Gurarij space and the Poulsen simplex are studied. The Gurarij space is a classical Banach space constructed by Gurarij [25] that can be characterized as the only approximately ultrahomogeneous separable Banach space whose age consists of all the finite-dimensional Banach spaces [4, 32]. It is proved in [3] that the group Aut $(\mathbb{G})$ of automorphisms of $\mathbb{G}$ is extremely amenable. Besides the KPT correspondence, the proof relies on an analysis of the structure of isometric embedding between finite-dimensional Banach spaces of the form $\ell_{\infty}^{n}$. Such embeddings are described in terms of rigid surjections between finite ordered sets. This makes it possible to apply the Dual Ramsey Theorem [22] to, ultimately, establish the (approximate) Ramsey property for the class of finite-dimensional Banach spaces.

A similar technique is employed in [3] to determined the universal minimal flow of the group Aut ( $\mathbb{P})$ of affine homeomorphisms of the Poulsen simplex [55]. The Poulsen simplex $\mathbb{P}$ is the unique Choquet simplex with the remarkable property that its boundary $\partial \mathbb{P}$ is dense within the simplex $\mathbb{P}$ itself-in stark contrast with what happens for the more common Bauer simplices, which have closed boundary. 
One can describe $\mathbb{P}$ in terms of homogeneous structures by means of the Kadison correspondence [1], which assigns to a Choquet simplex $K$ the function system $A(K)$ of continuous affine scalarvalued functions on $K$. This establishes an equivalence of categories between compact convex sets and function systems, where Choquet simplices correspond to nuclear function systems. The function system $A(\mathbb{P})$ corresponding to the Poulsen simplex is the only (nuclear) function system whose age is the class of all the finite-dimensional function systems. Relying on this correspondence, as well as the KPT correspondence and the Dual Ramsey Theorem, it is proved in [3] that $\mathbb{P}$ itself, regarded as an $\operatorname{Aut}(\mathbb{P})$-flow with the canonical action of Aut $(\mathbb{P})$, is universal (and minimal), whence it is the universal minimal Aut $(\mathbb{P})$-flow.

In this paper these results are extended to the noncommutative analogues of these objects, which can be constructed in the setting of operator spaces and operator systems. An operator space $X$ is a complex vector space endowed with a norm on $K(H) \otimes X$, where $B(H)$ is the algebra of operators on the separable infinite-dimensional Hilbert space $H$, and $K(H) \subseteq B(H)$ is the algebra of compact operators. Concretely, separable operator spaces can be thought of as closed subspaces $X$ of a $\mathrm{C}^{*}$ algebra or, equivalently, of $B(H)$, endowed with the norm induced by the inclusion $K(H) \otimes X \subseteq$ $B(H) \otimes B(H) \subseteq B(H \otimes H)$. Every Banach space can be regarded as an operator space, and the operator spaces that arise in this fashion are precisely those that can be realized as subspaces of commutative $\mathrm{C}^{*}$-algebras. The theory of operator spaces can be thought of as a noncommutative generalization of the theory of Banach spaces, and it has applications in the study of $\mathrm{C}^{*}$-algebras and quantum information theory [47].

In much the same way, function systems admit operator systems as noncommutative analogues. An operator system is an operator space that can be realized as a closed subspace $B(H)$ that is unital, in the sense that it contains the identity operator - the unit. (Naturally, in this context morphisms are also required to be unital, namely to map the unit to the unit.) Choquet simplices in turn correspond to the operator systems that are nuclear, which is an approximation property akin to amenability of groups or Banach algebras. In this context, the Kadison correspondence between function systems and compact convex sets can be generalized to a correspondence between compact matrix convex sets and operator systems, which we recall in Section 4.1. Operator systems can thus be thought of as noncommutative analogues of compact convex sets, and noncommutative Choquet theory in this context has been recently developed in [9] building on [11, 12, 14, 16, 17, 58]. Operator systems also arise in the study of operator algebras, nonlocal games, and free real algebraic geometry [27, 28].

The noncommutative Gurarij space $\mathbb{N} \mathbb{G}$ was constructed by Oikhberg [46] and can be characterized as the only (approximately) ultrahomogeneous separable nuclear operator space whose age contains all the operator spaces that can be realized as a subspace of a finite-dimensional $\mathrm{C}^{*}$-algebra [36]. In this paper, we prove that, as in the commutative case, the group Aut $(\mathbb{N} \mathbb{G})$ of automorphisms of $\mathbb{N} G$ is extremely amenable.

The noncommutative Poulsen simplex $\mathbb{N P}$ is a noncommutative Choquet simplex (compact matrix convex set) whose corresponding operator system $A(\mathbb{N P})$ of matrix-valued continuous affine functions is the unique separable nuclear operator system whose age contains all the operator systems that can be realized as unital subspaces of a finite-dimensional $\mathrm{C}^{*}$-algebra [37]. One can also characterize $A(\mathbb{N P})$ as the unique separable nuclear operator system that is universal in the sense of Kirchberg and Wassermann [31, 38]. This property can be though of as a noncommutative analogue of having dense extreme boundary. It is also true that the matrix extreme points of $\mathbb{N P}$ are dense in $\mathbb{N P}$, although it is 
unknown if this property characterizes $\mathbb{N P}$ among the metrizable noncommutative Choquet simplices. Due to the canonical role that $\mathbb{P}$ plays within the class of Choquet simplices (as, for instance, it contains any metrizable Choquet simplex as a proper face), it is natural to expect that $\mathbb{N P}$ will be an example of fundamental importance for noncommutative Choquet theory [9]. We prove in this paper that the compact space $\mathbb{N P}_{1}$ of 1-dimensional points of $\mathbb{N P}$ - which are precisely the unital linear functionals on $A(\mathbb{N P})$ - endowed with the canonical action of the group Aut $(\mathbb{N P})$ of matrix-affine homeomorphisms of $\mathbb{N P}$, is the universal minimal Aut $(\mathbb{N P})$-flow. This is the natural noncommutative analogue of the corresponding result for $\mathbb{P}$ from [3].

The paper is organized as follows. In Section 2 we review some fundamental notions concerning operator spaces and operator systems. We also define the notion of Fraïssé classes, Fraïssé limits, and the KPT correspondence restricted to this specific context. In Section 3, we introduce several nuclear operator spaces as Fraïssé limits of classes of finite dimensional injective operator spaces, and then in Section 3.2 we establish the (approximate) Ramsey property for each of these classes, obtaining a proof of extreme amenability of Aut $(\mathbb{N} \mathbb{G})$. In Section 4, we consider the more general case of structures consisting of an operator space with a distinguished morphism to another (fixed) operator space $R$. Finally, in Section 4 we consider noncommutative Choquet simplices, operator systems, operator systems with distinguished state, and we compute the universal minimal flow of Aut (NPP).

Acknowledgments. We are grateful to Itaï, Ben Yaacov, Clinton Conley, Valentin Ferenczi, Alexander Kechris, Matt Kennedy, Julien Melleray, Lionel Nguyen Van Thé, Vladimir Pestov, Slawomir Solecki, Stevo Todorcevic, and Todor Tsankov for several helpful conversations and remarks.

\section{Fraïssé Classes AND the RAMSEy PROPERTy of OPERATOR SPACES AND SYSTEMS}

2.1. Operator spaces and operator systems. We now recall some fundamental notions and results from the theory of operator spaces. The monographs $[6,13,54]$ provide good introductions to this subject. An operator space $E$ is a closed linear subspace of the space $B(H)$ of bounded linear operators on some complex Hilbert space $H$. The inclusion $E \subset B(H)$ induces matrix norms on each $M_{n}(E)$, $n \in \mathbb{N}$, the space of $n \times n$ matrices with entries in $E$. The norm of an element $\left[x_{i j}\right]$ of $M_{n}(E)$ is defined as the operator norm of $\left[x_{i j}\right]$ when regarded in the canonical way as an linear operator on the $n$-fold Hilbertian direct sum of $H$ by itself. The $\infty$-sum of two operator spaces $E \subset B\left(H_{0}\right)$ and $F \subset B\left(H_{1}\right)$ is the space $E \oplus_{\infty} F \subset B\left(H_{0} \oplus H_{1}\right)$ of operators of the form

$$
\left[\begin{array}{ll}
x & 0 \\
0 & y
\end{array}\right]
$$

for $x \in E$ and $y \in F$. One can equivalently define operator spaces as the closed subspaces of unital $\mathrm{C}^{*}$-algebras. A unital $\mathrm{C}^{*}$-algebra is a closed subalgebra of $B(H)$ containing the identity operator and closed under taking adjoints. Unital $\mathrm{C}^{*}$-algebras can be abstractly characterized as the complex Banach algebras with multiplicative identity and involution satisfying the $\mathrm{C}^{*}$-identity $\left\|a^{*} a\right\|=\|a\|^{2}$. Operator spaces also admit an abstract characterization, in terms of Ruan's axioms for the matrix norms [48, Theorem 13.4]. Precisely, a matrix normed complex vector space $X$ is an operator space if and only if the matrix norms satisfy the identity

$$
\left\|\alpha_{1}^{*} x_{1} \beta_{1}+\cdots+\alpha_{\ell}^{*} x_{\ell} \beta_{\ell}\right\| \leqslant\left\|\alpha_{1}^{*} \alpha_{1}+\cdots+\alpha_{\ell}^{*} \alpha_{\ell}\right\| \max \left\{\left\|x_{1}\right\|, \ldots,\left\|x_{\ell}\right\|\right\}\left\|\beta_{1}^{*} \beta_{1}+\cdots+\beta_{\ell}^{*} \beta_{\ell}\right\|
$$


for $n_{1}, \ldots, n_{\ell}, n \in \mathbb{N}, x_{i} \in M_{n_{i}}(X)$, and $\alpha_{i}, \beta_{i} \in M_{n_{i}, n}(\mathbb{C})$. In this identity, one consider the natural notion of matrix multiplication between matrices over $X$ and scalar matrices. The norm of scalar matrices is the operator norm, where matrices are regarded as operators on finite-dimensional Hilbert spaces.

The abstract characterization of $\mathrm{C}^{*}$-algebras shows that, whenever $K$ is a compact Hausdorff space, $C(K)$ with the pointwise operations and the supremum norm is a unital $\mathrm{C}^{*}$-algebra. The unital $\mathrm{C}^{*}$ algebras of this form are precisely the abelian ones. Any complex Banach space $E$ has a canonical operator space structure, obtained by representing $E$ isometrically as a subspace of $C\left(\operatorname{Ball}\left(E^{*}\right)\right)$, where the unit ball Ball $\left(E^{*}\right)$ of $E^{*}$ is endowed with the weak*-topology. This operator space structure on $E$ is called its minimal quantization [13] and the corresponding operator space is denoted by $\operatorname{MIN}(E)$. The matrix norms on $\operatorname{MIN}(E)$ are defined by $\left\|\left[x_{i j}\right]\right\|=\sup _{\phi \in \operatorname{Ball}\left(E^{*}\right)}\left\|\left[\phi\left(x_{i j}\right)\right]\right\|$ for $\left[x_{i j}\right] \in M_{n}(E)$. The operator spaces that arise in this fashion are called minimal operator spaces. These are precisely the operator spaces that can be represented inside an abelian unital $\mathrm{C}^{*}$-algebra. Arbitrary operator spaces can be thought of as the noncommutative analog of Banach spaces.

If $\phi: E \rightarrow F$ is a linear map between operator spaces, then one can consider its amplifications $\phi^{(n)}: M_{n}(E) \rightarrow M_{n}(F)$ obtained by applying $\phi$ entrywise. The completely bounded norm $\|\phi\|_{\mathrm{cb}}$ of $\phi$ is the supremum of $\left\|\phi^{(n)}\right\|$ for $n \in \mathbb{N}$. A linear map $\phi$ is completely bounded if $\|\phi\|_{\mathrm{cb}}$ is finite, and completely contractive if $\|\phi\|_{\mathrm{cb}}$ is at most 1 . The cb-distance between two completely bounded linear maps $\phi, \psi: E \rightarrow F$ is defined by $d_{\mathrm{cb}}(\phi, \psi):=\|\phi-\psi\|_{\mathrm{cb}}$. From now on, we regard the space of completely bounded maps from $E$ to $F$, and all its subspaces, as a metric space endowed with the cb-distance $d_{\mathrm{cb}}$.

We regard operator spaces as the objects of a category having completely contractive linear maps as morphisms. An isomorphism in this category is a surjective linear complete isometry, which is an invertible completely contractive linear map with completely contractive inverse. If $E$ is an operator space, then its automorphism group $\operatorname{Aut}(E)$ is the group of surjective linear complete isometries from $E$ to itself. When $E$ is separable, this is a Polish group when endowed with the topology of pointwise convergence. The dual operator space of an operator space $E$ is a canonical operator space structure on the space $E^{*}$ of (completely) bounded linear functionals on $E$, obtained by identifying completely isometrically $M_{n}\left(E^{*}\right)$ with the space of completely bounded linear maps from $E$ to $M_{n}(\mathbb{C})$; see $[13$, $\S 3.2]$.

When $E, F$ are Banach spaces, and $\phi: E \rightarrow F$ is a linear map, then $\phi$ is bounded if and only if it is completely bounded when $E$ and $F$ are endowed with their minimal operator space structure. Furthermore, in this case one has the equality of norms $\|\phi: E \rightarrow F\|=\|\phi: \operatorname{MIN}(E) \rightarrow \operatorname{MIN}(F)\|_{\mathrm{cb}}$. Thus the category of Banach spaces and contractive linear maps can be seen as a full subcategory of the category of operator spaces and completely contractive linear maps. In particular, the group of surjective linear isometries of a Banach space $E$ can be identified with the group of surjective linear complete isometries of $\operatorname{MIN}(E)$. We will identify a Banach space $E$ with the corresponding minimal operator space $\operatorname{MIN}(E)$.

There is a natural class of geometric objects that correspond to operator spaces, generalizing the correspondence between Banach spaces and compact absolutely convex sets. A compact rectangular matrix convex set in a topological vector space $V$ is a sequence $\left(K_{n, m}\right)$ of compact convex subsets of $M_{n, m}(V)$, the $n \times m$-matrices over $V$, endowed with a notion of rectangular convex combination. This is an expression $\alpha_{1}^{*} p_{1} \beta_{1}+\cdots+\alpha_{\ell}^{*} p_{\ell} \beta_{\ell}$ for $p_{i} \in K_{n_{i}, m_{i}}, \alpha_{i} \in M_{n_{i}, n}(\mathbb{C})$, and $\beta_{i} \in M_{m_{i}, m}(\mathbb{C})$ satisfying 
$\left\|\alpha_{1}^{*} \alpha_{1}+\cdots+\alpha_{\ell}^{*} \alpha_{\ell}\right\| \leqslant 1$ and $\left\|\beta_{1}^{*} \beta_{1}+\cdots+\beta_{\ell}^{*} \beta_{\ell}\right\| \leqslant 1$. The notion of an affine map and extreme points admit natural rectangular matrix analogs, where usual convex combinations are replaced with rectangular matrix convex combinations. When $E$ is an operator space, let $\mathrm{CBall}\left(E^{*}\right)$ be the sequence $\left(K_{n, m}\right)$, where each $K_{n, m}$ is the unit ball of $M_{n, m}\left(E^{*}\right)$. It is proved in [19] that any compact rectangular matrix convex set arises in this way. Furthermore the correspondence $E \mapsto \operatorname{CBall}\left(E^{*}\right)$ is a contravariant equivalence of categories from the category of operator spaces and completely contractive maps to the category of compact rectangular matrix convex sets and continuous rectangular affine maps.

An operator system is a closed linear subspace $X$ of the algebra $B(H)$ for some Hilbert space $H$ that is unital and self-adjoint, i.e. contains the identity operator and is closed under taking adjoints. In particular, the space $M_{n}(\mathbb{C})$ has a natural operator system structure, obtained by identifying $M_{n}(\mathbb{C})$ with $B\left(\ell_{2}^{n}\right)$. An operator system $X$ inherits from the inclusion $X \subset B(H)$ an involution $x \mapsto x^{*}$, which corresponds to taking adjoints, and a distinguished element 1 (the unit), which corresponds to the identity operator. Furthermore, for every $n \in \mathbb{N}, M_{n}(X)$ has a canonical norm and a notion of positivity, obtained by setting $\left[x_{i j}\right] \geqslant 0$ if and only if $\left[x_{i j}\right]$ is positive when regarded as an operator on the $n$-fold Hilbertian sum of $H$ by itself. The self-adjoint part $X_{\mathrm{sa}}$ of $X$ is the unital subspace of $X$ containing those $x \in X$ such that $x=x^{*}$. A linear map $\phi: X \rightarrow Y$ between operator systems is unital if it maps the unit of $X$ to the unit of $Y$, positive if it maps positive elements to positive elements, and completely positive if every amplification $\phi^{(n)}$ is positive. We abbreviate "unital completely positive linear map" as "ucp map". It is well known that a unital linear map $\phi$ between operator systems is completely positive if and only if it is completely contractive. A unital complete isometry $\phi: X \rightarrow Y$ is called a complete order embedding. A surjective complete order embedding is a complete order isomorphism. One can abstractly characterize the pairs $(X, 1)$, where $X$ is an operator space and $1 \in X$, that are operator systems, in the sense that there exists a complete isometry $\phi: X \rightarrow B(H)$ mapping 1 to the identity operator and $X$ onto a closed self-adjoint subspace of $B(H)$ [7]. An earlier abstract characterization of operator systems in terms of the matrix positive cones is due to Choi and Effros [8].

2.2. Fraïssé classes and Fraïssé limits. We recall in this section Fraïssé classes and Fraïssé limits in the setting of operator spaces and operator systems. These can be seen as particular instances of Fraïssé classes and Fraïssé limits of metric structures in the sense of [5, 44]. In order to make this paper more self-contained, we will introduce all these notions in this particular case.

Let 0 sp be the class of operator spaces. Given $X, Y \in 0$ sp and $\delta \geqslant 0$, let $\operatorname{Emb}_{\delta}^{0 \text { sp }}(X, Y)$ be the space of $\delta$-embeddings form $X$ into $Y$, that is, injective complete contractions $\phi: X \rightarrow Y$ such that $\left\|\phi^{-1}\right\|_{\mathrm{cb}} \leqslant 1+\delta$. In this terminology, the complete isometries are precisely the 0 -embeddings, which we will simply call embeddings. Let $\operatorname{Aut}^{\text {Osp }}(X)$ be the group of surjective embeddings from $X$ to itself. One can deduce from the small perturbation lemma in operator space theory [54, Lemma 2.13.2] that $\operatorname{Emb}_{\delta}^{0 \text { sp }}(X, Y)$ is a compact metric space whenever $X, Y$ are finite-dimensional objects of Osp. When $\delta=0$, we will write $\operatorname{Emb}^{0 \mathrm{sp}}(X, Y)$ instead of $\operatorname{Emb}_{0}^{0 \mathrm{sp}}(X, Y)$. The members of $\operatorname{Emb}^{0 \mathrm{sp}}(X, Y)$ are called 0sp-embeddings from $X$ into $Y$. We also have that, when $X$ is separable, $\operatorname{Aut}^{\text {osp }}(X)$ is a Polish group when endowed with the topology of pointwise convergence. Whenever there is no possibility of confusion we will use $\operatorname{Emb}_{\delta}(X, Y)$ and $\operatorname{Aut}(X)$ instead of $\operatorname{Emb}_{\delta}^{\text {Osp }}(X, Y)$ and $\operatorname{Aut}^{0 \text { sp }}(X)$, respectively.

Given an operator space $R$, by an $R$-operator space we mean a pair $\boldsymbol{X}=\left(X, s_{X}\right)$, where $X$ is an operator space and $s_{X}: X \rightarrow R$ is a complete contraction. Let $0 \mathrm{sp}^{R}$ be the collection of $R$-operator 
spaces. Given $\boldsymbol{X}=\left(X, s_{X}\right)$ and $\boldsymbol{Y}=\left(Y, s_{Y}\right)$ in $0 \mathrm{sp}^{R}$, and $\delta \geqslant 0$, let $\operatorname{Emb}_{\delta}^{0 \mathrm{sp}^{R}}(\boldsymbol{X}, \boldsymbol{Y})$ be the space of $\delta$-embeddings $\phi: X \rightarrow Y$ such that $\left\|s_{Y} \circ \phi-s_{X}\right\|_{\mathrm{cb}} \leqslant \delta$. This is a metric space when we consider the metric $d_{\mathrm{cb}}(\phi, \psi):=\|\phi-\psi\|_{\mathrm{cb}}$. Again, we will call a 0 -embedding, simply, an embedding. We let $\mathrm{Aut}^{0 \mathrm{sp}^{R}}(\boldsymbol{X})$ be the group of surjective linear complete isometries $\phi$ from $X$ to itself such that $s_{Y} \circ \phi=s_{X}$. Note that when $R$ is the trivial operator space $\{0\}, R$-operator spaces can be identified with operator spaces.

Similarly, we let 0sy be the class of operator systems. Given $X, Y \in 0$ sy, and $\delta \geqslant 0$, let $\operatorname{Emb}_{\delta}^{\text {osy }}(X, Y)$ be the collection of all injective unital complete contractions $\phi: X \rightarrow Y$ such that $\left\|\phi^{-1}\right\|_{\mathrm{cb}} \leqslant 1+\delta$. For a fixed operator system $R$, let $0 \mathrm{sy}^{R}$ be the class of $R$-operator systems, that is pairs $\boldsymbol{X}=\left(X, s_{X}\right)$ where $X$ is a operator system and $s_{X}: X \rightarrow R$ is an unital complete contraction. We define $\operatorname{Emb}_{\delta}^{\text {Osy }}(\boldsymbol{X}, \boldsymbol{Y})$ to be the collection of all injective unital complete contractions $\phi: X \rightarrow Y$ such that $\left\|\phi^{-1}\right\|_{\mathrm{cb}} \leqslant 1+\delta$ and $\left\|s_{X}-s_{Y} \circ \phi\right\|_{\mathrm{cb}} \leqslant \delta$, endowed with the metric $d_{\mathrm{cb}}(\phi, \psi):=\|\phi-\psi\|_{\mathrm{cb}}$. We also define $\mathrm{Aut}^{\mathrm{Dsy}}(X)$ to be the group of unital surjective complete isometries from $X$ to itself, and $\mathrm{Aut}^{0 \mathrm{sy}^{R}}(\boldsymbol{X})$ to be the group of unital surjective complete isometries $\phi$ from $X$ to itself such that $s_{X} \circ \phi=s_{X}$. Also, when $\boldsymbol{X}=\left(X, s_{X}\right), \boldsymbol{Y}=\left(Y, s_{Y}\right)$ are $R$-operator spaces or $R$-operator systems, we write $\boldsymbol{X} \subseteq \boldsymbol{Y}$ to denote that $X \subseteq Y$ and $s_{Y} \uparrow_{X}=s_{X}$.

In the next definitions, $\mathrm{C}$ is either $0 \mathrm{sp}, 0 \mathrm{sy}, 0 \mathrm{sp}^{R}$ for a fixed operator space $R$, or $0 \mathrm{sy}^{R}$ for a fixed operator system $R$.

Definition 2.1 (Gromov-Hausdorff pseudometric). The Gromov-Hausdorff pseudometric $d_{\mathrm{C}}$ is defined by setting, for finite-dimensional $X, Y \in \mathrm{C}, d_{\mathrm{C}}(X, Y)$ to be the infimum of all $\delta>0$ such that there exist $f \in \operatorname{Emb}_{\delta}^{\mathrm{C}}(X, Y)$ and $g \in \operatorname{Emb}_{\delta}^{\mathrm{C}}(Y, X)$ such that $\left\|g \circ f-\operatorname{Id}_{X}\right\|_{\mathrm{cb}}<\delta$ and $\left\|f \circ g-\operatorname{Id}_{Y}\right\|_{\mathrm{cb}}<\delta$.

It is worth to point out that when $\mathrm{C}$ is the class of operator spaces, then it is easily seen that for every finite dimensional operator systems $X$ and $Y$ one has that $d_{\mathrm{C}}(X, Y) \leqslant d_{\mathrm{BM}}(X, Y) \leqslant 3 d_{\mathrm{C}}(X, Y)$, where

$$
d_{\mathrm{BM}}(X, Y):=\log \left(\inf \left\{\|T\|_{\mathrm{cb}}\left\|T^{-1}\right\|_{\mathrm{cb}}: T: X \rightarrow Y \text { is a complete bounded isomorphism }\right\}\right)
$$

is the well known Banach-Mazur pseudometric. It follows that the class of operator spaces of dimension $n$ has diameter $\leqslant n$, although this class is not compact for $n \geqslant 3$. The reader can find more information in [54, Chapter 21].

In the following, we let $\varpi: \mathbb{R}^{+} \rightarrow \mathbb{R}^{+}$be a strictly increasing function, continuous at 0 , and vanishing at 0 , such that $\varpi(\delta) \geqslant \delta$.

Definition 2.2 (Stable Fraïssé class). Let $\mathcal{A} \subseteq \mathrm{C}$.

(a) $\mathcal{A}$ satisfies the stable amalgamation property (SAP) with modulus $\varpi$ when for every $X, Y, Z \in \mathcal{A}$, $\delta \geqslant 0, \varepsilon>0, \phi \in \operatorname{Emb}_{\delta}^{\mathrm{C}}(X, Y)$, and $\psi \in \operatorname{Emb}_{\delta}^{\mathrm{C}}(X, Z)$, there exist $V \in \mathcal{A}, i \in \operatorname{Emb}^{\mathrm{C}}(Y, V)$, and $j \in \operatorname{Emb}^{\mathrm{C}}(Z, V)$ such that $\|i \circ \phi-j \circ \psi\|_{\mathrm{cb}} \leqslant \varpi(\delta)+\varepsilon$.

(b) $\mathcal{A}$ is a stable amalgamation class with modulus $\varpi$ when $\mathcal{A}$ has the (SAP) and the joint embedding property, that is, for every $X, Y \in \mathcal{A}$ there exists $Z \in \mathcal{A}$ such that $\operatorname{Emb}^{\mathrm{C}}(X, Z)$ and $\operatorname{Emb}^{\mathrm{C}}(Y, Z)$ are nonempty.

(c) $\mathcal{A}$ is a stable Fraïssé class with modulus $\varpi$ when it is a stable amalgamation class and $\mathcal{A}$ is separable with respect to the Gromov-Hausdorff pseudometric $d_{C}$.

It is easy to see that if $\mathcal{A}$ has an element that can be embedded into any other member of $\mathcal{A}$ then the stable amalgamation property for $\mathcal{A}$ implies the joint embedding property for $\mathcal{A}$. 
Definition 2.3 (Stable Homogeneity). Let $\mathcal{A} \subseteq \mathrm{C}$. We say that $M \in \mathrm{C}$ satisfies the stable homogeneity property with respect to $\mathcal{A}$ with modulus $\varpi$ if:

(a) $\operatorname{Emb}^{\mathrm{C}}(X, M)$ is nonempty for every $X \in \mathcal{A}$.

(b) For every $X \in \mathcal{A}, \delta \geqslant 0, \varepsilon>0$, and $f, g \in \operatorname{Emb}_{\delta}^{\mathrm{C}}(X, M)$ there is $\alpha \in \operatorname{Aut}^{\mathrm{C}}(M)$ such that $\|\alpha \circ g-f\|_{\mathrm{cb}} \leqslant \varpi(\delta)+\varepsilon$.

When $\mathcal{A}$ is the collection of all finite-dimensional $X \subseteq M$ in $\mathrm{C}$, we say that $M$ is stably homogeneous with modulus $\varpi$.

Definition 2.4. Given a stable Fraïssé class $\mathcal{A} \subseteq \mathrm{C}$, we write $[\mathcal{A}]$ to denote the class of $E \in \mathrm{C}$ such that every finite-dimensional $X \subseteq E$ is a limit, with respect to the Gromov-Hausdorff distance, of a sequence of subspaces of elements in $\mathcal{A}$. Let $\langle\mathcal{A}\rangle$ be the collection of all finite-dimensional elements of $[\mathcal{A}]$.

Notice that $E \in[\mathcal{A}]$ if and only if for every finite-dimensional $X \subseteq E$ and every $\delta>0$ there is some $Y \in \mathcal{A}$ such that $\operatorname{Emb}_{\delta}^{\mathrm{C}}(X, Y) \neq \varnothing$.

Definition 2.5 (Fraïssé limit). Let $\mathcal{A} \subseteq$ C. The stable Fraïssé limit of $\mathcal{A}$ (with modulus $\varpi$ ), denoted by $\operatorname{FLim} \mathcal{A}$, if it exists, is the unique separable object in $[\mathcal{A}]$ that is $\mathcal{A}$-stably homogeneous (with modulus $\varpi)$.

A usual back-and-forth argument shows the following; see for instance [37, Subsection 2.6], [18, Theorem 2.26].

Proposition 2.6. Suppose that $\mathcal{A} \subseteq \mathrm{C}$ is a stable amalgamation class, and $M \in \mathrm{C}$ is separable. Then the Fraïssé limit FLim $\mathcal{A}$ exists. Furthermore the following assertions are equivalent:

(1) $M=\operatorname{FLim} \mathcal{A}$.

(2) $M$ is stably homogeneous with modulus $\varpi$, and the class $\operatorname{Age}^{\mathrm{C}}(M)$ of all finite-dimensional $X \in \mathrm{C}$ such that $X \subseteq M$ is equal to $\langle\mathcal{A}\rangle$.

Notice that whenever $\operatorname{FLim}\langle\mathcal{A}\rangle$ exists, $\mathrm{FLim} \mathcal{A}$ also exists and it must be equal to $\mathrm{FLim}\langle\mathcal{A}\rangle$. Stable Fraïssé classes and stable Fraïssé limits are in particular Fraïssé classes and Fraïssé limits as metric structures in the sense of [4]. One can realize $\mathrm{FLim} \mathcal{A}$ as the limit of an inductive sequence of elements of $\mathcal{A}$ with C-embeddings as connective maps, and it can be proved that every separable structure in $[\mathcal{A}]$ admits a C-embedding into FLim $\mathcal{A}$.

The nomenclature "homogeneous" is related to the concept of "disposition" in Banach space theory, that for example was used by V. I. Gurarij in [25] to define his space (of universal "placement"). At a midpoint of both notions, we say that $M \in \mathrm{C}$ is stably of $\mathcal{A}$-disposition (with modulus $\varpi$ ) when $\operatorname{Emb}^{\mathrm{C}}(X, M) \neq \varnothing$ for every $X \in \mathcal{A}$ and when for every $\delta \geqslant 0$ and $\varepsilon>0$, every $X, Y \in \mathcal{A}$ every $f \in \operatorname{Emb}_{\delta}^{\mathrm{C}}(X, M)$ and $\iota \in \operatorname{Emb}_{\delta}^{\mathrm{C}}(X, Y)$ there is some $g \in \operatorname{Emb}^{\mathrm{C}}(Y, M)$ such that $\|g \circ \iota-f\|_{\mathrm{cb}} \leqslant \varpi(\delta)+\varepsilon$. It it proved in [37, Proposition 2.12], implication $(6) \Rightarrow(1)$, that a separable $M \in[\mathcal{A}]$ is the stable Fraïssé limit of $\mathcal{A}$ if and only if $M$ is stably of $\mathcal{A}$-disposition.

Several structures in functional analysis arise as the Fraïssé limit of a suitable class $\mathcal{A}$. For example the class of finite-dimensional operator Hilbert spaces is a Fraïssé class, and its corresponding limit is the separable operator Hilbert space $\mathrm{OH}$ introduced and studied in [53]. Another natural example of a family with the stable amalgamation property is the collection of finite-dimensional Banach spaces $\left\{\ell_{p}^{n}\right\}_{n \geqslant 0}$ for every $p \in(1,+\infty)$. In the case $p=2$ one can use the polar decomposition for bounded 
operators on a Hilbert space to deduce that every $\delta$-embedding between Hilbert spaces is close to an embedding. The other cases are treated in [18]. In this case one uses a result by Schechtman in [56] stating that for every such $p \neq 2$ there exists a function $\varpi_{p}: \mathbb{R}_{+} \rightarrow \mathbb{R}_{+}$continuous at 0 and vanishing at 0 , with the property that if $\phi: \ell_{p}^{k} \rightarrow \ell_{p}^{m}$ is a $\delta$-embedding for some $\delta>0$, then there exist $n \in \mathbb{N}, I \in \operatorname{Emb}\left(\ell_{p}^{m}, \ell_{p}^{n}\right)$, and $J \in \operatorname{Emb}\left(\ell_{p}^{k}, \ell_{p}^{n}\right)$ such that $\|J-I \circ \phi\| \leqslant \varpi_{p}(\delta)$. The corresponding Fraïssé $\operatorname{limit} \operatorname{FLim}\left\{\ell_{p}^{n}\right\}_{n}$ of $\left\{\ell_{p}^{n}\right\}_{n}$ is the Lebesgue space $L_{p}[0,1]$. When $p$ is an even integer other than 2 , the space $L_{p}[0,1]$ is not stably homogeneous or, equivalently, the class $\left\langle\left\{\ell_{p}^{n}\right\}_{n}\right\rangle$ does not have the stable amalgamation property. In fact, in this case, $L_{p}[0,1]$ is not even approximately ultrahomogeneous as shown in [41]; see also [18]. An operator space $M$ is approximately ultrahomogeneous when for every finite-dimensional $X \subseteq M$, every complete isometry $\phi: X \rightarrow M$, and every $\varepsilon>0$ there is a surjective linear complete isometry $\alpha: M \rightarrow M$ such that $\left\|\alpha \uparrow_{X}-\phi\right\|_{\mathrm{cb}} \leqslant \varepsilon$. Obviously, stably homogeneous spaces are approximately ultrahomogeneous. Lusky proved in [41] that the space $L_{p}[0,1]$ is approximately ultrahomogeneous when $p \in(1,+\infty)$ is not an even integer. This has been recently improved on [18, Theorem 4.1] where it is shown that the spaces $L_{p}[0,1]$ for those $p$ 's are "quasi" stably homogeneous in the sense that there is a modulus of stability depending on dimensions $\widetilde{\varpi}: \mathbb{N} \times[0, \infty) \rightarrow[0, \infty)$ such that for every $\delta \geqslant 0, \varepsilon>0$, every finite dimensional subspace $X \subseteq L_{p}[0,1]$, and $f, g \in \operatorname{Emb}_{\delta}\left(X, L_{p}[0,1]\right)$ there exists $\alpha \in \operatorname{Aut}\left(L_{p}[0,1]\right)$ such that $\|\alpha \circ g-f\|_{\mathrm{cb}} \leqslant \varpi(\operatorname{dim} X, \delta)+\varepsilon$.

The Ramsey property of the following classes, proved to be Fraïssé in [37], are the main subject of the present paper. Recall that an operator space $X$ is injective if for every operator spaces $Y \subseteq Z$, every complete contraction from $Y$ to $X$ can be extended to a complete contraction from $Z$ to $X$. One defines injective operator systems similarly, by replacing complete contractions with unital complete contractions.

Theorem 2.7. Let $\mathrm{C}$ be either 0sp or 0sy. Suppose that $\mathcal{I}$ is a countable class of finite-dimensional injective elements of $\mathrm{C}$ such that for $X, Y \in \mathcal{I}$, the $\infty$-sum $X \oplus_{\infty} Y$ embeds into an element of $\mathcal{I}$. Then $\mathcal{I}$ is a stable amalgamation class and $\langle\mathcal{I}\rangle$ is a stable Fraïssé class, both with stability modulus $\varpi(\delta)=\delta$ if $\mathrm{C}$ is 0sp, and $\varpi(\delta)=2 \delta$ if $\mathrm{C}$ is 0 sy.

For a class $\mathcal{A}$ of operator spaces, and an operator space $R$, we let $\mathcal{A}^{R}$ be the class of $R$-operator spaces of the form $(X, s)$ where $X \in \mathcal{A}$. There is a close relation between the stable amalgamation property of $\mathcal{A}$ and the one of $\mathcal{A}^{R}$. Similar considerations and notation apply in the case of operator systems.

Proposition 2.8. Let $\mathrm{C}$ be either 0sp or 0sy. Suppose that $\mathcal{A}$ is a class of finite-dimensional injective elements of $\mathrm{C}$ that has the stable amalgamation property with modulus $\varpi(\delta)$. Suppose that $R \in \mathrm{C}$ is separable and such that every element in $\operatorname{Age}^{\mathrm{C}}(R)$ embeds into an element of $\mathcal{A}$.

If either: (a) for $X, Y \in \mathcal{A}$, the $\infty$-sum $X \oplus_{\infty} Y$ belongs to $\mathcal{A}$, or (b) $R$ is injective, and for $X, Y \in \mathcal{A}$, the $\infty$-sum $X \oplus_{\infty} Y$ has a $\mathrm{C}$-embedding into an element of $\mathcal{A}$, then the class $\mathcal{A}^{R}$ satisfies the stable amalgamation property with modulus $\varpi(\delta)+\delta$.

Moreover, suppose that each element of $\mathcal{A}$ is injective, and $R$ is $\mathcal{A}$-nuclear, that is, the identity map of $R$ is the pointwise limit of C-morphisms that factor through elements of $\mathcal{A}$; see [37, Definition 3.4]. Then the Fraïssé limit $\mathrm{FLim} \mathcal{A}^{R}$ is $(\mathrm{FLim} \mathcal{A}, \Omega)$ for an appropriate complete contraction $\Omega$ : $\mathrm{FLim} \mathcal{A} \rightarrow R$, that when $\mathrm{C}=0$ sy is in addition unital (see Proposition 3.12 (3) and Proposition 4.4 (3) for more details). 
Proof. We suppose that (a) holds. The proof when (b) holds is similar. Let $\delta \geqslant 0, \varepsilon>0, \boldsymbol{X}=\left(X, s_{X}\right)$, $\boldsymbol{Y}=\left(Y, s_{Y}\right)$ and $\boldsymbol{Z}=\left(Z, s_{Z}\right)$ all in $\mathcal{A}^{R}$, and let $\phi \in \operatorname{Emb}_{\delta}^{\mathrm{C}^{R}}(\mathbf{X}, \mathbf{Z})$ and $\psi \in \operatorname{Emb}_{\delta}^{\mathrm{C}^{R}}(\mathbf{X}, \mathbf{Y})$. By definition, $\phi \in \operatorname{Emb}_{\delta}^{\mathrm{C}}(X, Z)$ and $\psi \in \operatorname{Emb}_{\delta}^{\mathrm{C}}(X, Y)$, so it follows from the (SAP) of the class $\mathcal{A}$ with modulus $\varpi$ that there is some $V \in \mathcal{A}, i \in \operatorname{Emb}^{\mathrm{C}}(Y, V)$ and $j \in \operatorname{Emb}^{\mathrm{C}}(Z, V)$ such that $\|i \circ \phi-j \circ \psi\|_{\mathrm{cb}} \leqslant \varpi(\delta)+\varepsilon$. Let $R_{0} \in \mathcal{A}$, and let $\theta \in \operatorname{Emb}^{\mathrm{C}}\left(S, R_{0}\right)$ where $S \in \operatorname{Age}^{\mathrm{C}}(R)$ is generated by $\operatorname{Im} s_{Y}+\operatorname{Im} s_{Z}$. Set, $W:=$ $V \oplus_{\infty} R_{0} \in \mathcal{A}$. We define $\mathbf{W}:=\left(W, \pi_{2}\right) \in \mathcal{A}^{R}$, where $\pi_{2}: V \oplus_{\infty} R_{0} \rightarrow R_{0} \subseteq R$ is the canonical secondcoordinate projection. Let also $I: Y \rightarrow W$ and $J: Z \rightarrow W$ be defined by $I(y):=\left(i(y), \theta\left(s_{Y}(y)\right)\right)$ and $J(z):=\left(j(z), \theta\left(s_{Z}(z)\right)\right)$. It follows that $I \in \operatorname{Emb}^{\mathrm{C}^{R}}(\mathbf{Y}, \mathbf{W})$ and that $J \in \operatorname{Emb}^{\mathrm{C}^{R}}(\mathbf{Z}, \mathbf{W})$. From definitions, we have that $\|I \circ \phi-J \circ \psi\|_{\mathrm{cb}}=\max \left\{\|i \circ \phi-j \circ \psi\|_{\mathrm{cb}},\left\|\theta \circ s_{Y} \circ \phi-\theta \circ s_{Z} \circ \psi\right\|_{\mathrm{cb}}\right\} \leqslant$ $\max \left\{\varpi(\delta)+\varepsilon,\left\|s_{Y} \circ \phi-s_{X}\right\|_{\mathrm{cb}}+\left\|s_{Z} \circ \psi-s_{X}\right\|_{\mathrm{cb}}\right\} \leqslant \varpi(\delta)+\delta+\varepsilon$, as we are assuming that $\varpi$ satisfies $\varpi(\delta) \geqslant \delta$.

Suppose now that each element of $\mathcal{A}$ is injective, and that $R$ is $\mathcal{A}$-nuclear. We consider the case of operator spaces. The case of operator systems is entirely similar. Write FLim $\mathcal{A}^{R}=(\mathbb{M}, \Omega)$. We now show that $\mathbb{M}=\operatorname{FLim} \mathcal{A}$. To this purpose, as we mentioned before, it suffices to prove that the following property of approximate local disposition holds: for every $\varepsilon>0, \delta \geqslant 0, F \in \mathcal{A}$, tuple $\bar{a}=\left(a_{0}, \ldots, a_{n}\right)$ in $F$, injective completely contractive map $f: \operatorname{span}(\bar{a}) \rightarrow \mathbb{M}$ such that $\left\|f^{-1}\right\|_{\mathrm{cb}} \leqslant \delta$, there exists a completely isometric linear map $g: F \rightarrow \mathbb{M}$ such that $\max _{i}\left\|g\left(a_{i}\right)-f\left(a_{i}\right)\right\| \leqslant \varpi(\delta)+\delta+\varepsilon$.

Consider the completely contractive map $\Omega \circ f: \operatorname{span}(\bar{a}) \rightarrow R$. We are assuming that $R$ is $\mathcal{A}$-nuclear. By the equivalence of $(1)$ and (3) in [37, Proposition 3.5], there exists a completely contractive map $s: F \rightarrow R$ such that $\max _{i}\left\|s\left(a_{i}\right)-(\Omega \circ f)\left(a_{i}\right)\right\| \leqslant \varepsilon$. By the homogeneity property of FLim $\mathcal{A}^{R}=$ $(\mathbb{M}, \Omega)$, there exists a $C^{R}$-embedding $g:(F, s) \rightarrow(\mathbb{M}, \omega)$ such that $\left\|g\left(a_{i}\right)-f\left(a_{i}\right)\right\| \leqslant \varpi(\delta)+\delta+\varepsilon$. This concludes the proof.

2.3. The approximate Ramsey property. We now introduce and characterize various version of the Ramsey property, which are strengthening of the amalgamation property and that, as we will see, can be used to obtain information about the automorphism group of Fraïssé limits. We still adopt the notation from above.

Definition 2.9 (Approximate and stable Ramsey Property). Let C be one of the classes Osp, Osp ${ }^{R}$, Osy or $0 \mathrm{sy}^{R}$, and let $\mathcal{A} \subseteq \mathrm{C}$.

(a) $\mathcal{A}$ satisfies the approximate Ramsey property (ARP) if for every $X, Y \in \mathcal{A}, \varepsilon>0$ there exists $Z \in \mathcal{A}$ such that any continuous coloring of $\operatorname{Emb}^{\mathrm{C}}(X, Z) \varepsilon$-stabilizes on $\gamma \circ \operatorname{Emb}^{\mathrm{C}}(X, Y)$ for some $\gamma \in \operatorname{Emb}^{\mathrm{C}}(Y, Z)$; that is, for every 1-Lipschitz mapping $c: \operatorname{Emb}^{\mathrm{C}}(X, Z) \rightarrow[0,1]$ there is some $\gamma \in \operatorname{Emb}^{\mathrm{C}}(Y, Z)$ such that $\operatorname{Osc}\left(c \uparrow\left(\gamma \circ \operatorname{Emb}^{\mathrm{C}}(X, Y)\right)\right)=\max _{\eta_{0}, \eta_{1} \in \operatorname{Emb}^{\mathrm{c}}(X, Y)}\left|c\left(\gamma \circ \eta_{0}\right)-c\left(\gamma \circ \eta_{1}\right)\right| \leqslant \varepsilon$.

(b) $\mathcal{A}$ satisfies the stable Ramsey property (SRP) with stability modulus $\varpi$ if for every $X, Y \in \mathcal{A}, \varepsilon>0$, $\delta \geqslant 0$ there exists $Z \in \mathcal{A}$ such that every continuous coloring of $\operatorname{Emb}_{\delta}^{\mathrm{C}}(X, Z)(\varepsilon+\varpi(\delta))$-stabilizes on $\gamma \circ \operatorname{Emb}_{\delta}^{\mathrm{C}}(X, Y)$ for some $\gamma \in \operatorname{Emb}^{\mathrm{C}}(Y, Z)$.

(c) $\mathcal{A}$ satisfies the discrete approximate Ramsey property, or discrete (ARP), if for every $X, Y \in$ $\mathcal{A}, r \in \mathbb{N}$ and $\varepsilon>0$ there exists $Z \in \mathcal{A}$ such that every $r$-coloring of $\operatorname{Emb}^{\mathrm{C}}(X, Z)$ has an $\varepsilon$ monochromatic set of the form $\gamma \circ \operatorname{Emb}^{\mathrm{C}}(X, Y)$ for some $\gamma \in \operatorname{Emb}^{\mathrm{C}}(Y, Z)$; that is, for every coloring $c: \operatorname{Emb}^{\mathrm{C}}(X, Z) \rightarrow r=\{0,1, \ldots, r-1\}$ there is some $\gamma \in \operatorname{Emb}^{\mathrm{C}}(Y, Z)$ and some $j \in r$ such that $\gamma \circ \operatorname{Emb}^{\mathrm{C}}(X, Y) \subseteq\left(c^{-1}(j)\right)_{\varepsilon}$. The discrete stable Ramsey property, or discrete (SRP), is defined similarly. 
(d) The compact (ARP) and the compact (SRP) of $\mathcal{A}$ are defined as the (ARP) and the (SRP), respectively, by replacing continuous colorings with compact colorings, i.e. 1-Lipschitz mappings into compact metric spaces.

It is not difficult to see that the (SRP) with modulus $\varpi$ of a class implies the stable amalgamation property of the class with modulus $\varpi$. Also, it is worth to point out that the (ARP) as in Definition 2.9 is equivalent to the one considered in [44, Definition 3.3] when $R$-operator spaces or systems are regarded as structures in the logic for metric structures [5] as in [21, Appendix B] or [37, §8.1]. The following proposition provides reformulations of the (ARP) in terms of discrete or compact colorings, and it is a generalization of [3, Proposition 2.7] where the case of Banach spaces is treated.

Proposition 2.10. Let $\mathrm{C}$ be one of the classes $0 \mathrm{sp}, 0 \mathrm{sp}^{R}$, Osy or $0 \mathrm{sy}^{R}$. The following are equivalent for a class $\mathcal{A} \subseteq \mathrm{C}$ :

(1) $\mathcal{A}$ satisfies the $(A R P)$.

(2) $\mathcal{A}$ satisfies the discrete $(A R P)$.

(3) $\mathcal{A}$ satisfies the compact $(A R P)$.

Proof. The compact (ARP) obviously implies the (ARP). Suppose that $\mathcal{A}$ satisfies the (ARP), and let us prove that $\mathcal{A}$ satisfies the discrete (ARP). This is done by induction on $r \in \mathbb{N}$. The case $r=1$ is trivial. Suppose that we have shown that $\mathcal{A}$ satisfies the discrete (ARP) for $r$-colorings. Consider $X, Y \in \mathcal{A}$ and $\varepsilon>0$. Then by the inductive hypothesis, there is $Z_{0} \in \mathcal{A}$ such that every $r$-coloring of $\operatorname{Emb}^{\mathrm{C}}\left(X, Z_{0}\right) \varepsilon$-stabilizes on $\gamma \circ \operatorname{Emb}^{\mathrm{C}}(X, Y)$ for some $\gamma \in \operatorname{Emb}^{\mathrm{C}}\left(Y, Z_{0}\right)$. Since by the assumption $\mathcal{A}$ satisfies the (ARP), there is $Z \in \mathcal{A}$ such that every continuous coloring of $\operatorname{Emb}^{\mathrm{C}}(X, Z) \varepsilon / 2$-stabilizes on $\gamma \circ \operatorname{Emb}^{\mathrm{C}}\left(X, Z_{0}\right)$ for some $\gamma \in \operatorname{Emb}^{\mathrm{C}}\left(Z_{0}, Z\right)$. We claim that $Z$ witnesses that $\mathcal{A}$ satisfies the discrete $(\mathrm{ARP})$ for $(r+1)$-colorings. Indeed, suppose that $c$ is an $(r+1)$-coloring of $\operatorname{Emb}^{\mathrm{C}}(X, Z)$. Define $f: \operatorname{Emb}^{\mathrm{C}}(X, Z) \rightarrow[0,1]$ by $f(\phi):=\frac{1}{2} d_{\mathrm{cb}}\left(\phi, c^{-1}(r)\right)$. This is a continuous coloring, so by the choice of $Z$ there exists $\gamma \in \operatorname{Emb}^{\mathrm{C}}\left(Z_{0}, Z\right)$ such that $f \varepsilon / 2$-stabilizes on $\gamma \circ \operatorname{Emb}^{\mathrm{C}}\left(X, Z_{0}\right)$. Now, if there is some $\phi \in \operatorname{Emb}^{\mathrm{C}}\left(X, Z_{0}\right)$ such that $c(\gamma \circ \phi)=r$, then $\gamma \circ \operatorname{Emb}^{\mathrm{C}}\left(X, Z_{0}\right) \subseteq\left(c^{-1}(r)\right)_{\varepsilon}$, so choosing an arbitrary $\bar{\gamma} \in \operatorname{Emb}^{\mathrm{C}}\left(Y, Z_{0}\right)$ we obtain that $c \varepsilon$-stabilizes on $\gamma \circ \bar{\gamma} \circ \operatorname{Emb}^{\mathrm{C}}(X, Y)$. Otherwise, $\left(\gamma \circ \operatorname{Emb}^{\mathrm{C}}\left(X, Z_{0}\right)\right) \cap c^{-1}(r)=\varnothing$, so defining $\bar{c}(\phi):=c(\gamma \circ \phi)$ for $\phi \in \operatorname{Emb}^{\mathrm{C}}\left(X, Z_{0}\right)$ gives an $r$ coloring of $\operatorname{Emb}^{\mathrm{C}}\left(X, Z_{0}\right)$. By the choice of $Z_{0}$ there exists $\bar{\gamma} \in \operatorname{Emb}^{\mathrm{C}}\left(Y, Z_{0}\right)$ such that $\bar{c} \varepsilon$-stabilizes on $\bar{\gamma} \circ \operatorname{Emb}^{\mathrm{C}}(X, Y)$. Therefore $c \varepsilon$-stabilizes on $\gamma \circ \bar{\gamma} \circ \operatorname{Emb}^{\mathrm{C}}(X, Y)$. This concludes the proof that the (ARP) implies the discrete (ARP).

Finally, the discrete (ARP) implies the compact (ARP). Indeed, given $\varepsilon>0$ and a compact metric space $K$, one can find a finite $\varepsilon$-dense subset $D \subseteq K$. Thus if $Z \in \mathcal{A}$ witnesses the discrete (ARP) for $X, Y, \varepsilon$ and $D$, then given a 1-Lipschitz $f: \operatorname{Emb}^{\mathrm{C}} \rightarrow K$ we can define a coloring $c: \operatorname{Emb}^{\mathrm{C}}(X, Z) \rightarrow$ $D \subseteq K$ such that $d_{K}(c(\phi), f(\phi)) \leqslant \varepsilon$ for every $\phi \in \operatorname{Emb}^{\mathrm{C}}(X, Z)$. In this way, if $c \varepsilon$-stabilizes on $\gamma \circ \operatorname{Emb}^{\mathrm{C}}(X, Y)$, then $f 3 \varepsilon$-stabilizes on $\gamma \circ \operatorname{Emb}^{\mathrm{C}}(X, Y)$.

The following is a useful property of classes with the (SAP). It can be easily proved by induction, using the fact that the $\operatorname{spaces}^{\operatorname{Emb}_{\delta}}(X, Y)$ for finite-dimensional $X, Y \in \mathcal{A}$ are compact (See also [3, Claim 2.13.2], where a similar result is proved for Banach spaces).

Proposition 2.11. Suppose that $\mathcal{A}$ satisfies the stable amalgamation property with modulus $\varpi$. For every $\mathcal{F} \subseteq \mathcal{A}$ finite and every $\varepsilon, \delta>0$ there exist $V \in \mathcal{A}$ and $I_{X} \in \operatorname{Emb}^{\mathrm{C}}(X, V)$ for $X \in \mathcal{F}$ such that 
for every (not necessarily distinct) $X, Y, Z \in \mathcal{F}$ and every $\gamma \in \operatorname{Emb}_{\delta}^{\mathrm{C}}(X, Y)$ and $\eta \in \operatorname{Emb}_{\delta}^{\mathrm{C}}(X, Z)$ there exists $J \in \mathrm{Emb}^{\mathrm{C}}(Z, V)$ such that $\left\|I_{Y} \circ \gamma-J \circ \eta\right\|_{\mathrm{cb}} \leqslant \varpi(\delta)+\varepsilon$.

In particular, for every $X, Y \in \mathcal{F}$ one has that $I_{Y} \circ \operatorname{Emb}_{\delta}^{\mathrm{C}}(X, Y) \subseteq\left(\operatorname{Emb}^{\mathrm{C}}(X, V)\right)_{\varpi(\delta)+\varepsilon}$.

The next proposition generalizes [3, Proposition 2.13].

Proposition 2.12. Suppose that $\mathcal{A} \subseteq \mathrm{C}$ satisfies the stable amalgamation property with modulus $\varpi$. Then the following assertions are equivalent:

(1) $\mathcal{A}$ satisfies the (ARP).

(2) $\mathcal{A}$ satisfies the (SRP) with modulus $\varpi$.

(3) $\mathcal{A}$ satisfies the discrete (SRP) with modulus $\varpi$.

(4) $\mathcal{A}$ satisfies the compact (SRP) with modulus $\varpi$.

Suppose that $\mathcal{A} \subseteq \mathcal{B} \subseteq\langle\mathcal{A}\rangle$ also satisfies the stable amalgamation property with modulus $\varpi$. Then (1)-(4) are equivalent to any of the following conditions.

(5) $\mathcal{B}$ satisfies the (ARP).

(6) For every $X, Y \in \mathcal{A}$, every $\varepsilon>0$ and every $r \in \mathbb{N}$ there is $Z \in \mathcal{B}$ such that every $r$-coloring of $\operatorname{Emb}^{\mathrm{C}}(X, Z)$ has an $\varepsilon$-monochromatic set of the form $\gamma \circ \operatorname{Emb}^{\mathrm{C}}(X, Y)$ for some $\gamma \in \operatorname{Emb}^{\mathrm{C}}(Y, Z)$.

Proof. A simple modification of the proof of the Proposition 2.10 gives that the compact (SRP) with modulus $\varpi$ implies the (SRP) with modulus $\varpi$, which in turn implies the discrete (SRP) with modulus $\varpi$. Trivially, the discrete (SRP) with modulus $\varpi$ implies the discrete (ARP), and this one is equivalent to the (ARP) by Proposition 2.10. We will now show that the (ARP) implies the compact (SRP) with modulus $\varpi$. Suppose that $\mathcal{A}$ satisfies the (ARP). Fix $X, Y \in \mathcal{A}, \delta, \varepsilon>0$ and a compact metric space $K$. We use Proposition 2.11 to find $Y_{0} \in \mathcal{A}$ such that for every $\phi, \psi \in \operatorname{Emb}_{\delta}^{\mathrm{C}}(X, Y)$ there are $i, j \in \operatorname{Emb}^{\mathrm{C}}\left(Y, Y_{0}\right)$ such that $\|i \circ \phi-j \circ \psi\|_{\mathrm{cb}} \leqslant \varpi(\delta)+\varepsilon$. We will consider the space $\operatorname{Lip}\left(\operatorname{Emb}_{\delta}^{\mathrm{C}}(X, Y), K\right)$ of 1-Lipschitz maps from $\operatorname{Emb}_{\delta}^{\mathrm{C}}(X, Y)$ to $K$ as a compact metric space, endowed with the metric $d(f, g)=\sup \left\{d_{K}(f(\phi), g(\phi)): \phi \in \operatorname{Emb}_{\delta}^{\mathrm{C}}(X, Y)\right\}$. By Proposition 2.10, $\mathcal{A}$ satisfies the compact (ARP), so we apply it to $Y, Y_{0} \in \mathcal{A}$ and the compact space $\operatorname{Lip}\left(\operatorname{Emb}_{\delta}^{\mathrm{C}}(X, Y), K\right)$, and obtain some $Z \in \mathcal{A}$ such that every 1-Lipschitz coloring $c: \operatorname{Emb}^{\mathrm{C}}(Y, Z) \rightarrow \operatorname{Lip}\left(\operatorname{Emb}_{\delta}^{\mathrm{C}}(X, Y), K\right) \varepsilon$-stabilizes on $\gamma \circ \operatorname{Emb}^{\mathrm{C}}\left(Y, Y_{0}\right)$ for some $\gamma \in \operatorname{Emb}^{\mathrm{C}}\left(Y_{0}, Z\right)$. We claim that $Z$ works, so let $c: \operatorname{Emb}_{\delta}^{\mathrm{C}}(X, Z) \rightarrow K$ be 1-Lipschitz. We can then define the 1-Lipschitz mapping $\widehat{c}: \operatorname{Emb}^{\mathrm{C}}(Y, Z) \rightarrow \operatorname{Lip}\left(\operatorname{Emb}_{\delta}^{\mathrm{C}}(X, Y), K\right)$ by setting, for $\gamma \in \operatorname{Emb}^{\mathrm{C}}(Y, Z), \widehat{c}(\gamma): \operatorname{Emb}_{\delta}^{\mathrm{C}}(X, Y) \rightarrow K, \phi \mapsto c(\gamma \circ \phi)$. By the choice of $Z$, there exists $\bar{\gamma} \in \operatorname{Emb}^{\mathbb{C}}\left(Y_{0}, Z\right)$ be such that $\widehat{c} \varepsilon$-stabilizes on $\bar{\gamma} \circ \operatorname{Emb}^{\mathbb{C}}\left(Y, Y_{0}\right)$. Choose an arbitrary $\varrho \in \operatorname{Emb}^{\mathrm{C}}\left(Y, Y_{0}\right)$. We claim that $c(3 \varepsilon+\varpi(\delta))$-stabilizes on $\bar{\gamma} \circ \varrho \circ \operatorname{Emb}_{\delta}^{\mathrm{C}}(X, Y)$. Let $\phi, \psi \in \operatorname{Emb}_{\delta}^{\mathrm{C}}(X, Y)$. By the choice of $Y_{0}$ there are $i, j \in \operatorname{Emb}^{\mathrm{C}}\left(Y, Y_{0}\right)$ such that $d_{\mathrm{cb}}(i \circ \phi, j \circ \psi) \leqslant \varepsilon+\varpi(\delta)$. Since $d(\widehat{c}(\bar{\gamma} \circ \varrho), \widehat{c}(\bar{\gamma} \circ i)) \leqslant \varepsilon$ and $d(\widehat{c}(\bar{\gamma} \circ \varrho), \widehat{c}(\bar{\gamma} \circ j)) \leqslant \varepsilon$, it follows that $d_{K}(c(\bar{\gamma} \circ \varrho \circ \phi), c(\bar{\gamma} \circ i \circ \phi)) \leqslant \varepsilon, d_{K}(c(\bar{\gamma} \circ \varrho \circ \psi), c(\bar{\gamma} \circ j \circ \psi)) \leqslant \varepsilon$. Furthermore, from $d_{\mathrm{cb}}(i \circ \phi, j \circ \psi) \leqslant \varepsilon+\varpi(\delta)$ and the fact that $c$ is 1-Lipschitz we deduce that $d_{K}(c(\bar{\gamma} \circ \varrho \circ \phi), c(\bar{\gamma} \circ \varrho \circ \psi)) \leqslant 3 \varepsilon+\varpi(\delta)$.

Suppose now that $\mathcal{A} \subseteq \mathcal{B} \subseteq\langle\mathcal{A}\rangle$ also satisfies the stable amalgamation property with the same modulus $\varpi$. Obviously (1) implies (6). Let us prove that (6) implies (5): We prove that, assuming that (6) holds, the class $\mathcal{B}$ satisfies the discrete (ARP). Fix $X, Y \in \mathcal{B}$.

Claim 2.12.1. Let $\varepsilon, \delta>0$ and let $X_{0} \in \mathcal{A}$ and $t_{X} \in \operatorname{Emb}_{\delta}^{\mathrm{C}}\left(X, X_{0}\right)$. There are $Y_{0} \in \mathcal{A}$ and $t_{Y} \in$ $\operatorname{Emb}_{\delta}^{\mathrm{C}}\left(Y, Y_{0}\right)$ such that for every $\eta \in \operatorname{Emb}^{\mathrm{C}}(X, Y)$ there is some $\gamma \in \operatorname{Emb}^{\mathrm{C}}\left(X_{0}, Y_{0}\right)$ such that $\| t_{Y} \circ \eta-$ $\gamma \circ t_{X} \|_{\mathrm{cb}} \leqslant \varpi(\delta)+\varepsilon$. 
Proof of Claim: Let $0<\delta_{0}<\delta$ be such that $\varpi\left(\delta_{0}\right) \leqslant \varpi(\delta) / 3$ and set $\varepsilon_{0}=\varepsilon / 3$. Let $Z_{0} \in \mathcal{A}$ and $t_{0} \in \operatorname{Emb}_{\delta_{0}}^{\mathrm{C}}\left(Y, Z_{0}\right)$. Apply Proposition 2.11 in the case of the class $\mathcal{B}$ to $\mathcal{F}:=\left\{X, X_{0}, Z_{0}\right\}, \delta_{0}$ and $\varepsilon_{0}$ to obtain $Z_{1} \in \mathcal{B}$ and $J_{0} \in \operatorname{Emb}^{\mathrm{C}}\left(Z_{0}, Z_{1}\right)$. Fix $Z_{2} \in \mathcal{A}$ and $t_{1} \in \operatorname{Emb}_{\delta_{0}}^{\mathrm{C}}\left(Z_{1}, Z_{2}\right)$. Apply again Proposition 2.11, now in the case of the class $\mathcal{A}$, for $\mathcal{F}:=\left\{X_{0}, Z_{0}, Z_{2}\right\}, \delta_{0}$ and $\varepsilon_{0}$ to find the $Y_{0} \in \mathcal{A}$, and $J_{1} \in \operatorname{Emb}^{\mathrm{C}}\left(Z_{2}, Y_{0}\right)$. Let $I \in \operatorname{Emb}^{\mathrm{C}}\left(Z_{0}, Y_{0}\right)$ be such that

$$
\left\|J_{1} \circ t_{1} \circ J_{0}-I\right\|_{\mathrm{cb}} \leqslant \varpi\left(\delta_{0}\right)+\varepsilon_{0} .
$$

We claim that $Y_{0}$ and $t_{Y}:=I \circ t_{0} \in \operatorname{Emb}_{\delta_{0}}^{\mathrm{C}}\left(Y, Y_{0}\right) \subseteq \operatorname{Emb}_{\delta}^{\mathrm{C}}\left(Y, Y_{0}\right)$ work. Suppose that $\eta \in \operatorname{Emb}^{\mathrm{C}}(X, Y)$. By the choice of $Z_{1}$ there is some $\hat{\eta} \in \mathrm{Emb}^{\mathrm{C}}\left(X_{0}, Z_{1}\right)$ such that

$$
\left\|J_{0} \circ t_{0} \circ \eta-\hat{\eta} \circ t_{X}\right\|_{\mathrm{cb}} \leqslant \varpi\left(\delta_{0}\right)+\varepsilon_{0} .
$$

Now by the choice of $Y_{0}$, there is some $\gamma \in \operatorname{Emb}^{\mathrm{C}}\left(X_{0}, Y_{0}\right)$ such that

$$
\left\|J_{1} \circ t_{1} \circ \hat{\eta}-\gamma\right\|_{\mathrm{cb}} \leqslant \varpi\left(\delta_{0}\right)+\varepsilon_{0} .
$$

It follows from the inequalities in (1), (2) and (3),

$$
\begin{aligned}
\left\|I \circ t_{0} \circ \eta-J_{1} \circ t_{1} \circ J_{0} \circ t_{0} \circ \eta\right\|_{\mathrm{cb}} & \leqslant\left(\varpi\left(\delta_{0}\right)+\varepsilon_{0}\right)\left\|t_{0} \circ \eta\right\|_{\mathrm{cb}} \leqslant\left(\varpi\left(\delta_{0}\right)+\varepsilon_{0}\right), \\
\left\|J_{1} \circ t_{1} \circ J_{0} \circ t_{0} \circ \eta-J_{1} \circ t_{1} \circ \hat{\eta} \circ t_{X}\right\|_{\mathrm{cb}} & \leqslant\left\|J_{1} \circ t_{1}\right\|_{\mathrm{cb}}\left(\varpi\left(\delta_{0}\right)+\varepsilon_{0}\right) \leqslant\left(\varpi\left(\delta_{0}\right)+\varepsilon_{0}\right), \\
\left\|J_{1} \circ t_{1} \circ \hat{\eta} \circ t_{X}-\gamma \circ t_{X}\right\|_{\mathrm{cb}} & \leqslant\left(\varpi\left(\delta_{0}\right)+\varepsilon_{0}\right)\left\|t_{X}\right\|_{\mathrm{cb}} \leqslant\left(\varpi\left(\delta_{0}\right)+\varepsilon_{0}\right) .
\end{aligned}
$$

Consequently,

$$
\left\|t_{Y} \circ \eta-\gamma \circ t_{X}\right\|_{\mathrm{cb}}=\left\|I \circ t_{0} \circ \eta-\gamma \circ t_{X}\right\|_{\mathrm{cb}} \leqslant 3 \varpi\left(\delta_{0}\right)+3 \varepsilon_{0} \leqslant \varpi(\delta)+\varepsilon .
$$

Fix now $\varepsilon>0$, and let $\delta>0$ be such that $\varpi(\delta) \leqslant \varepsilon / 15$ and set $\varepsilon_{0}:=\varepsilon / 5$. Fix also $X_{0} \in \mathcal{A}$ and $t_{X} \in \operatorname{Emb}_{\delta}^{\mathrm{C}}\left(X, X_{0}\right)$. We use the Claim 2.12.1 to find $Y_{0} \in \mathcal{A}$ and $t_{Y} \in \operatorname{Emb}_{\delta}^{\mathrm{C}}\left(Y, Y_{0}\right)$. We apply (6) to $X_{0}, Y_{0}, \varepsilon_{0}$ and $r$ to find the corresponding $Z_{0} \in \mathcal{B}$. Since $\mathcal{B}$ satisfies the stable amalgamation property with modulus $\varpi$, we can apply Proposition 2.11 to $\mathcal{F}:=\left\{X_{0}, Y_{0}, X, Y, Z_{0}\right\}, \delta$ and $\varepsilon_{0}$ and find $Z \in \mathcal{B}$ and for each $W \in \mathcal{F}$ some $J_{W} \in \operatorname{Emb}^{\mathrm{C}}(W, Z)$ such that for every (not necessarily distinct) $W_{0}, W_{1}, W_{2} \in \mathcal{F}$ and every $\gamma \in \operatorname{Emb}_{\delta}^{\mathrm{C}}\left(W_{0}, W_{1}\right)$ and $\eta \in \operatorname{Emb}_{\delta}^{\mathrm{C}}\left(W_{0}, W_{2}\right)$ there is $J \in \operatorname{Emb}^{\mathrm{C}}\left(W_{2}, Z\right)$ such that $\left\|I_{W_{1}} \circ \gamma-J \circ \eta\right\|_{\mathrm{cb}} \leqslant \varpi(\delta)+\varepsilon_{0}$. We claim that $Z$ works. For suppose that $c: \operatorname{Emb}^{\mathrm{C}}(X, Z) \rightarrow r$, and we induce a coloring $\widehat{c}: \operatorname{Emb}^{\mathrm{C}}\left(X_{0}, Z_{0}\right) \rightarrow r$ as follows. By applying the defining property of $Z$ to the triple $\left(X, Z_{0}, X\right)$, we obtain that $I_{Z_{0}} \circ \operatorname{Emb}_{\delta}^{\mathrm{C}}\left(X, Z_{0}\right) \subseteq\left(\operatorname{Emb}^{\mathrm{C}}(X, Z)\right)_{\varpi(\delta)+\varepsilon_{0}}$, so, for each $\gamma \in \operatorname{Emb}^{\mathrm{C}}\left(X_{0}, Z_{0}\right)$ we can find $\hat{\gamma} \in \operatorname{Emb}^{\mathrm{C}}(X, Z)$ such that $\left\|I_{Z_{0}} \circ \gamma \circ t_{X}-\hat{\gamma}\right\|_{\mathrm{cb}} \leqslant \varpi(\delta)+\varepsilon_{0}$, and declare that $\widehat{c}(\gamma):=c(\hat{\gamma})$. Let $J \in \operatorname{Emb}^{\mathrm{C}}\left(Y_{0}, Z_{0}\right)$ and $j<r$ be such that $J \circ \operatorname{Emb}^{\mathrm{C}}\left(X_{0}, Y_{0}\right) \subseteq\left(\widehat{c}^{-1}(j)\right)_{\varepsilon_{0}}$. Let $I \in \operatorname{Emb}^{\mathrm{C}}(Y, Z)$ be such that

$$
\left\|I-I_{Z_{0}} \circ J \circ t_{Y}\right\|_{\mathrm{cb}} \leqslant \varpi(\delta)+\varepsilon_{0} .
$$

Let us see that $I \circ \operatorname{Emb}^{\mathrm{C}}(X, Y) \subseteq\left(c^{-1}(j)\right)_{\varepsilon}$. Fix $\eta \in \operatorname{Emb}^{\mathrm{C}}(X, Y)$. Let $\hat{\eta} \in \operatorname{Emb}^{\mathrm{C}}\left(X_{0}, Y_{0}\right)$ be such that

$$
\left\|\widehat{\eta} \circ t_{X}-t_{Y} \circ \eta\right\|_{\mathrm{cb}} \leqslant \varpi(\delta)+\varepsilon_{0} .
$$

Let $\gamma \in \operatorname{Emb}^{\mathrm{C}}\left(X_{0}, Z_{0}\right)$ be such that

$$
\|\gamma-J \circ \widehat{\eta}\|_{\mathrm{cb}} \leqslant \varepsilon_{0} \text { and } \widehat{c}(\gamma)=j
$$

so, by the definition of $\widehat{c}$, let now $\hat{\gamma} \in \operatorname{Emb}^{\mathrm{C}}(X, Z)$ be such that

$$
\left\|\hat{\gamma}-I_{Z_{0}} \circ \gamma \circ t_{X}\right\|_{\mathrm{cb}} \leqslant \varpi(\delta)+\varepsilon_{0} \text { and } c(\hat{\gamma})=j .
$$


Putting all this together,

$$
\begin{array}{r}
\left\|I \circ \gamma-I_{Z_{0}} \circ J \circ t_{Y} \circ \eta\right\|_{\mathrm{cb}} \leqslant \varpi(\delta)+\varepsilon_{0} \\
\left\|I_{Z_{0}} \circ J \circ t_{Y} \circ \eta-I_{Z_{0}} \circ J \circ \hat{\eta} \circ t_{X}\right\|_{\mathrm{cb}} \leqslant \varpi(\delta)+\varepsilon_{0} \\
\left\|I_{Z_{0}} \circ J \circ \hat{\eta} \circ t_{X}-I_{Z_{0}} \circ \gamma \circ t_{X}\right\|_{\mathrm{cb}} \leqslant \varepsilon_{0} .
\end{array}
$$

From (4), (5), (6) and (7) we obtain

$$
\|I \circ \eta-\hat{\gamma}\|_{\mathrm{cb}} \leqslant 3 \varpi(\delta)+4 \varepsilon_{0} \leqslant \varepsilon \text { and } c(\hat{\gamma})=j .
$$

(5) implies (1): The proof of that the discrete (ARP) of $\mathcal{B}$ implies the discrete (ARP) of $\mathcal{A}$ is similar to that of the implication 6 ) implies 5) so we only sketch it and we leave further details to the reader. Fix $X, Y \in \mathcal{A}, \varepsilon>0$ and $r \in \mathbb{N}$. Let $\delta>0$ be such that $\varpi(\delta) \leqslant \varepsilon / 8$ and set $\varepsilon_{0}:=\varepsilon / 4$. Let $Z_{0} \in \mathcal{B} \subseteq\langle\mathcal{A}\rangle$ be such that every $r$-coloring of $\operatorname{Emb}^{\mathrm{C}}\left(X, Z_{0}\right)$ has an $\varepsilon_{0}$-monochromatic set of the form $J \circ \operatorname{Emb}^{\mathrm{C}}(X, Y)$ for some $J \in \operatorname{Emb}^{\mathrm{C}}\left(Y, Z_{0}\right)$. Let $Z_{1} \in \mathcal{A}$ and $t \in \operatorname{Emb}_{\delta}^{\mathrm{C}}\left(Z_{0}, Z_{1}\right)$. We use the Proposition 2.11 in the case of the class $\mathcal{A}$ for $\mathcal{F}=\left\{X, Y, Z_{1}\right\}, \varepsilon$ and $\delta$ to find the corresponding $Z$. Then it follows that every $r$-coloring of $\mathrm{Emb}^{\mathrm{C}}(X, Z)$ has an $\varepsilon$-monochromatic set of the form $I \circ \operatorname{Emb}^{\mathrm{C}}(X, Y)$ for some $I \in \operatorname{Emb}^{\mathrm{C}}(Y, Z)$.

It is unclear to us whether the characterization of the (ARP) provided in 2.12 holds for an arbitrary class $\mathcal{A} \subseteq \mathrm{C}$.

Just like for the stable amalgamation property we presented in Proposition 2.8, we have the following relationship between the approximate Ramsey properties of a class $\mathcal{A}$ of f.d. operator spaces or systems, and the corresponding class $\mathcal{A}^{R}$.

Proposition 2.13. Let $\mathrm{C}$ be either 0sp or 0sy. Suppose that $\mathcal{A}$ is a class of finite-dimensional elements of $\mathrm{C}$ such that for $X, Y \in \mathcal{A}$, the $\infty$-sum $X \oplus_{\infty} Y$ belongs to $\mathcal{A}$.

(1) Suppose that $R \in \mathrm{C}$ is such that every element in $\operatorname{Age}^{\mathrm{C}}(R)$ embeds into an element of $\mathcal{A}$.

(1.1) If $\mathcal{A}$ has the (ARP), then the class $\mathcal{A}^{R}$ also has the (ARP).

(1.2) If $\mathcal{A}$ has the (SRP) with modulus $\varpi(\delta)$, then the class $\mathcal{A}^{R}$ also has the (SRP) with modulus $\varpi(\delta)+\delta$.

(2) Suppose that $R \in \mathcal{A}$.

(2.1) If $\mathcal{A}^{R}$ has the (ARP), then the class $\mathcal{A}$ also has the (ARP).

(2.2) If $\mathcal{A}^{R}$ has the (SRP) with modulus $\varpi(\delta)$, then the class $\mathcal{A}$ also has the (SRP) with modulus $\varpi(\delta)$.

Proof. (1.1) is proved similarly than (1) in Proposition 2.8, so we leave the details to the reader. (1.2): If $\mathcal{A}$ has the (SRP) with modulus $\varpi(\delta)$, then it satisfies the stable amalgamation property with modulus $\varpi(\delta)$. It follows from Proposition 2.8 that $\mathcal{A}^{R}$ satisfies the stable amalgamation property with modulus $\varpi(\delta)+\delta$, and from this, (1.1), and the equivalence between (1) and (2) in Proposition 2.12 , we obtain that $\mathcal{A}^{R}$ satisfies the (SRP) with modulus $\varpi(\delta)+\delta$. (2) is proved similarly.

2.3.1. Extreme amenability and Ramsey properties. Recall that a topological group $G$ is called extremely amenable if every continuous action of $G$ on a compact Hausdorff space has a fixed point. We are going to present a characterization of extreme amenability for automorphism groups of Fraïssé limits in one of the categories introduced before in terms of a Ramsey property, which can be seen as an instance of the Kechris-Pestov-Todorcevic correspondence [30] in the case of metric structures in 
[44]. The key for this characterization is the following intrinsic reformulation of extreme amenability from [51, Theorem 2.1.11].

Theorem 2.14. [51] A topological group $G$ is extremely amenable if and only if there is a directed collection of bounded left-invariant continuous pseudometrics $\left(d_{i}\right)_{i \in I}$ determining the topology of $G$ and such that every metric $G$-space $G /\left\{d_{i}=0\right\}$ is finitely oscillation stable, that is for every finite $F \subseteq G /\left\{d_{i}=0\right\}$, every bounded and uniformly continuous $\gamma: G /\left\{d_{i}=0\right\} \rightarrow \mathbb{R}$ and every $\varepsilon>0$ there is $g \in G$ such that $\operatorname{Osc}(\gamma \uparrow(g \cdot F))=\max _{\left[h_{0}\right],\left[h_{1}\right] \in F}\left|\gamma\left(\left[g \cdot h_{0}\right]\right)-\gamma\left(\left[g \cdot h_{1}\right]\right)\right| \leqslant \varepsilon$.

In the previous statement, $[h]$ denotes the equivalence class of $h$ in the quotient $G /\left\{d_{i}=0\right\}$. As before, let $\mathrm{C}$ be one of the classes $0 \mathrm{sp}, 0 \mathrm{sp}^{R}$, Osy, or $0 \mathrm{sy}^{R}$, with the corresponding notion of C-embedding. Fix $E \in \mathrm{C}$. For a fixed finite dimensional $X \subseteq E$, we let $d_{X}$ be the pseudometric on $\operatorname{Aut}^{\mathrm{C}}(E)$ defined by $d_{X}(\alpha, \beta):=\|(\alpha-\beta) \uparrow X\|_{\mathrm{cb}}$. In general, $\left(d_{X}\right)$ where $X$ varies within the finite-dimensional subspaces of $E$ defines the topology on $\operatorname{Aut}^{\mathrm{C}}(E)$. Suppose that $E \in \mathrm{C}$ is stably homogeneous. Observe that by the stable homogeneity property of $E$, the restriction map $\alpha \mapsto \alpha \uparrow X$ is an isometry from $\left(\operatorname{Aut}^{\mathrm{C}}(E), d_{X}\right)$ onto a dense subset of $\mathrm{Emb}^{\mathrm{C}}(X, E)$. In particular, a continuous coloring of $\left(\operatorname{Aut}^{\mathrm{C}}(E), d_{X}\right)$ induces a continuous coloring of $\operatorname{Emb}^{\mathrm{C}}(X, E)$. Also, given a finite $F \subseteq$ $\operatorname{Emb}^{\mathrm{C}}(X, E)$ we can find a finite dimensional $Y \subseteq E$ such that $F \subseteq \operatorname{Emb}^{\mathrm{C}}(X, Y)$, where we consider $\mathrm{Emb}^{\mathrm{C}}(X, Y)$ canonically included in $\operatorname{Emb}^{\mathrm{C}}(X, E)$ after composing with the corresponding inclusion of $Y$ into $E$. Reciprocally each set $\operatorname{Emb}^{\mathrm{C}}(X, Y)$ is compact, so it has finite $\varepsilon$-dense subsets. Theorem 2.14 can be restated as follows:

Lemma 2.15. The following statements are equivalent:

(a) $\operatorname{Aut}^{\mathrm{C}}(E)$ is extremely amenable.

(b) For every finite-dimensional $X, Y \subseteq E$ and every $\varepsilon>0$, every 2-coloring of $\operatorname{Emb}^{\mathrm{C}}(X, E)$, has an $\varepsilon$-monochromatic set of the form $\alpha \circ \operatorname{Emb}^{\mathrm{C}}(X, Y)$ for some $\alpha \in \operatorname{Aut}^{\mathrm{C}}(E)$, that is for every $c: \operatorname{Emb}^{\mathrm{C}}(X, E) \rightarrow r$ there is there exists $\alpha \in \operatorname{Aut}^{\mathrm{C}}(E)$ and $j \in\{0, \ldots, r-1\}$ such that for every $\gamma \in \operatorname{Emb}^{\mathrm{C}}(X, Y)$ there exists $\xi \in \operatorname{Emb}^{\mathrm{C}}(X, E)$ such that $c(\xi)=j$ and $\|\alpha \circ \gamma-\xi\|_{\mathrm{cb}} \leqslant \varepsilon$.

We refer the reader to [51, Chapter 1] for more information on oscillation stability. We suppose that $\mathcal{A} \subseteq \mathrm{C}$ is such that $\langle\mathcal{A}\rangle$ is a stable Fraïssé class with modulus $\varpi$, whose Fraïssé limit is $E$. We can now state the analogue in this context of the celebrated Kechris-Pestov-Todorcevic correspondence from [30]. The possibility of using the KPT correspondence in the setting of metric structures was first suggested by Melleray and Tsankov in [44].

Proposition 2.16 (KPT correspondence for $R$-operator spaces and systems). Suppose that $\mathcal{A} \subseteq \mathrm{C} i s$ such that $\mathcal{A}$ and $\langle\mathcal{A}\rangle$ satisfy the stable amalgamation property with stability modulus $\varpi$, and let $E$ be the corresponding Fraïssé limit. The following assertions are equivalent:

(1) $\operatorname{Aut}^{\mathrm{C}}(E)$ is extremely amenable.

(2) For every finite-dimensional $X, Y \subset E$ in $\mathrm{C}$ and $\delta \geqslant 0, \varepsilon>0$, every compact coloring of $\operatorname{Emb}_{\delta}^{\mathrm{C}}(X, E)$ has an $(\varepsilon+\varpi(\delta))$-monochromatic set of the form $\alpha \circ \operatorname{Emb}_{\delta}^{\mathrm{C}}(X, Y)$ for some $\alpha \in$ $\operatorname{Aut}^{\mathrm{C}}(E)$.

(3) For every finite-dimensional $X, Y \subset E$ in $\mathrm{C}$ and $\varepsilon>0$, every finite coloring of $\operatorname{Emb}^{\mathrm{C}}(X, E)$ has an $\varepsilon$-monochromatic set of the form $\alpha \circ \operatorname{Emb}^{\mathrm{C}}(X, Y)$ for some $\alpha \in \operatorname{Aut}^{\mathrm{C}}(E)$.

(4) For every $X, Y \subset E$ that belong to $\mathcal{A}$ and $\varepsilon>0$, every finite coloring of $\operatorname{Emb}^{\mathrm{C}}(X, E)$ has an $\varepsilon$-monochromatic set of the form $\alpha \circ \operatorname{Emb}^{\mathrm{C}}(X, Y)$ for some $\alpha \in \operatorname{Aut}^{\mathrm{C}}(E)$. 
(5) $\langle\mathcal{A}\rangle$ satisfies the $(A R P)$.

(6) $\mathcal{A}$ satisfies the $(A R P)$.

(7) For every $X, Y \in \mathcal{A}$, every $r \in \mathbb{N}$ and every $\varepsilon>0$ there is $Z \in\langle\mathcal{A}\rangle$ such that every $r$-coloring of $\operatorname{Emb}^{\mathrm{C}}(X, Z)$ has an $\varepsilon$-monochromatic set of the form $\gamma \circ \operatorname{Emb}^{\mathrm{C}}(X, Y)$ for some $\gamma \in \operatorname{Emb}^{\mathrm{C}}(Y, Z)$.

The key part is the equivalence between (1) and (7) since in this last condition, $X, Y \subseteq E$ are not arbitrary finite-dimensional subspaces, but are assumed to be in $\mathcal{A}$ while $Z$ is not necessarily in $\mathcal{A}$ but in the wider class $\langle\mathcal{A}\rangle$.

Proof. The proof uses standard arguments; see for example [44, Proposition 3.4 and Theorem 3.10]. We first show that properties (1)-(4) are equivalent, and then we show that properties (4)-(7) are equivalent.

(1) implies (2): Fix all data, in particular, let $c: \operatorname{Emb}_{\delta}^{\mathrm{C}}(X, E) \rightarrow K$ be a compact coloring, and let $K=\bigcup_{j<r} U_{j}$ be a covering of $K$ by disjoint sets $U_{j}$ of diameter less than $\varepsilon / 3$. We consider the induced coloring $\widehat{c}: \operatorname{Emb}^{\mathrm{C}}(X, E) \rightarrow r$ that declares $\widehat{c}(\gamma)=j$ when $c(\gamma) \in U_{j}$. Since $E$ is stably homogeneous with modulus $\varpi$, it follows from the Fraïssé correspondence in Theorem 2.6 that $\operatorname{Age}^{\mathrm{C}}(E)$ is a stable Fraïssé class with modulus $\varpi$, so we can use (1) in Proposition 2.11 to find $Y_{0} \subseteq E$ containing and $I \in \operatorname{Emb}\left(Y, Y_{0}\right)$ such that $I \circ \operatorname{Emb}_{\delta}^{\mathrm{C}}(X, Y) \subseteq\left(\operatorname{Emb}^{\mathrm{C}}\left(X, Y_{0}\right)\right)_{\varpi(\delta)+\varepsilon / 6}$. By composing with the corresponding inclusion, we identify each $\delta$-embedding from $X$ into $Y_{0}$ as a $\delta$-embedding from $X$ into $E$. Since $\operatorname{Emb}_{\delta}^{\mathrm{C}}\left(X, Y_{0}\right)$ is compact, we can use Lemma 2.15 to find $\beta \in \operatorname{Aut}^{\mathrm{C}}(E)$ and $j<r$ such that $\beta \circ \operatorname{Emb}_{\delta}^{\mathrm{C}}\left(X, Y_{0}\right) \subseteq\left(\widehat{c}^{-1}(j)\right)_{\varepsilon / 6}$. Let us see that $\alpha:=\beta \circ I$ satisfies the desired property: Given $\gamma \in \operatorname{Emb}_{\delta}^{\mathrm{C}}(X, Y)$, let $\eta \in \operatorname{Emb}^{\mathrm{C}}\left(X, Y_{0}\right)$ such that $\|I \circ \gamma-\eta\|_{\mathrm{cb}} \leqslant \varpi(\delta)+\varepsilon / 6$. Let also $\xi \in \operatorname{Emb}^{\mathrm{C}}(X, E)$ such that $c(\xi)=j$ and $\|\beta \circ \eta-\xi\|_{\mathrm{cb}} \leqslant \varepsilon / 6$, and consequently, $\|\alpha \circ \gamma-\xi\|_{\mathrm{cb}} \leqslant \varpi(\delta)+\varepsilon / 3$. Since $U_{j} \subseteq K$ has diameter less than $\varepsilon / 3$, it follows that $\operatorname{Osc}\left(c\left\lceil\left(\alpha \circ \operatorname{Emb}_{\delta}^{\mathrm{C}}(X, Y)\right)\right) \leqslant \varpi(\delta)+\varepsilon\right.$.

(2) implies (3): It suffices to prove that the following holds for every $\delta>0$ : for every finite-dimensional $X, Y \subset E$ in $\mathrm{C}$, and $\varepsilon>0$, every finite coloring of $\operatorname{Emb}_{\delta}^{\mathrm{C}}(X, E)$ has an $(\varepsilon+\varpi(\delta))$-monochromatic set of the form $\alpha \circ \operatorname{Emb}^{\mathrm{C}}(X, Y)$ for some $\alpha \in \operatorname{Aut}^{\mathrm{C}}(E)$. Fix a finite coloring $c: \operatorname{Emb}_{\delta}^{\mathrm{C}}(X, E) \rightarrow r$, and let $K$ be the ball of $\ell_{\infty}^{k}$ centered at 0 and of radius 2, and let $f: \operatorname{Emb}_{\delta}^{\mathrm{C}}(X, E) \rightarrow K$ be defined by $f(\sigma):=\left(d_{\mathrm{cb}}\left(\sigma, c^{-1}(i)\right)\right)_{i<r}$. This is a compact coloring, so by the hypothesis, there is $\alpha \in \operatorname{Aut}^{\mathrm{C}}(E)$ such that the oscillation of $f$ on $\alpha \circ \operatorname{Emb}_{\delta}^{\mathrm{C}}(X, Y)$ is at most $\varepsilon+\varpi(\delta)$. Then $\alpha \circ \operatorname{Emb}_{\delta}^{\mathrm{C}}(X, Y)$ is $(\varepsilon+\varpi(\delta))$ monochromatic for $c$. Indeed, fix $\bar{\phi} \in \operatorname{Emb}_{\delta}^{\mathrm{C}}(X, Y)$, and let $i:=c(\alpha \circ \bar{\phi})$. Then the $i$-th coordinate of $f(\alpha \circ \bar{\phi})$ with respect to the canonical basis of $\ell_{r}^{\infty}$ is zero. Since the oscillation of $f$ on $\alpha \circ \operatorname{Emb}_{\delta}^{\mathrm{C}}(X, Y)$ is at most $\varepsilon+\varpi(\delta)$, we have that for every $\phi \in \operatorname{Emb}_{\delta}^{\mathrm{C}}(X, Y), f(\alpha \circ \phi)$ and $f(\alpha \circ \bar{\phi})$ have distance at most $\varepsilon+\varpi(\delta)$. Since the $i$-th coordinate of $f(\alpha \circ \phi)$ is equal to $d_{\mathrm{cb}}\left(\alpha \circ \phi, c^{-1}(i)\right)$, this implies that $\left.\alpha \circ \phi, c^{-1}(i)\right) \leqslant \varepsilon+\varpi(\delta)$.

(3) implies (4) trivially.

(4) implies (7): It is easy to see that (4) and (7), respectively, are equivalent to

(4') for every $X, Y \in \mathcal{A}$, every compact metric space $\left(K, d_{K}\right), \varepsilon>0$ and 1-Lipschitz mapping $c$ : $\left(\mathrm{Emb}^{\mathrm{C}}(X, E), d_{\mathrm{cb}}\right) \rightarrow\left(K, d_{K}\right)$ there is some $\alpha \in \operatorname{Aut}^{\mathrm{C}}(E) \operatorname{such}$ that $\operatorname{Osc}\left(c \uparrow\left(\alpha \circ \operatorname{Emb}^{\mathrm{C}}(X, Y)\right)\right) \leqslant \varepsilon$.

$\left(7^{\prime}\right)$ for every $X, Y \in \mathcal{A}$, every compact metric space $\left(K, d_{K}\right)$ and $\varepsilon>0$ there is some $Z \in\langle\mathcal{A}\rangle$ such that for everyn 1-Lipschitz mapping $c: \operatorname{Emb}^{\mathrm{C}}(X, Z) \rightarrow K$ there is some $\gamma \in \operatorname{Emb}^{\mathrm{C}}(Y, Z)$ such that $\operatorname{Osc}\left(c \uparrow\left(\gamma \circ \operatorname{Emb}^{\mathrm{C}}(X, Y)\right)\right) \leqslant \varepsilon$.

This is similarly proved as the equivalence between the discrete (ARP) and the compact (ARP) (Proposition 2.10). Suppose by contradiction that ( $\left(^{\prime}\right)$ does not hold. Therefore there exist $X, Y \subset E$ 
in $\mathcal{A}, \varepsilon_{0}>0$ and a compact metric $\left(K, d_{K}\right)$ witnessing that. Let $D_{X} \subseteq \operatorname{Emb}^{\mathrm{C}}(X, E)$ and $D_{Y} \subseteq$ $\operatorname{Emb}^{\mathrm{C}}(Y, E)$ be countable dense subsets, and let $\left(Z_{n}\right)_{n \in \mathbb{N}}$ be an increasing sequence of finite-dimensional $Z_{n} \subset E$ in $\langle\mathcal{A}\rangle$, such that $Y \subseteq Z_{0}$ and such that for every $\phi \in D_{X}$ and $\psi \in D_{Y}$ there exists $n \in \mathbb{N}$ such that $\phi \in \operatorname{Emb}^{\mathrm{C}}\left(X, Z_{n}\right)$ and $\psi \in \operatorname{Emb}^{\mathrm{C}}\left(Y, X_{n}\right)$. This implies that $\bigcup_{n} \operatorname{Emb}^{\mathrm{C}}\left(X, Z_{n}\right)$ and $\bigcup_{n} \operatorname{Emb}^{\mathrm{C}}\left(Y, Z_{n}\right)$ are dense in $\operatorname{Emb}^{\mathrm{C}}(X, E)$ and $\operatorname{Emb}^{\mathrm{C}}(Y, E)$, respectively. Choose for each $n \in \mathbb{N}$ a "bad 1-Lipschitz coloring $c_{n}: \operatorname{Emb}^{\mathrm{C}}\left(X, Z_{n}\right) \rightarrow K$. Let $\mathcal{U}$ be a non-principal ultrafilter on $\mathbb{N}$. We define $c$ : $\operatorname{Emb}^{\mathrm{C}}(X, E) \rightarrow K$ by choosing for a given $\phi \in \operatorname{Emb}^{\mathrm{C}}(X, E)$ a sequence $\left(\phi_{n}\right)_{n}$, each $\phi_{n} \in \operatorname{Emb}^{\mathrm{C}}\left(X, Z_{n}\right)$, such that $\lim _{n} d_{\mathrm{cb}}\left(\phi, \phi_{n}\right)=0$, and then by declaring $c(\phi):=\mathcal{U}-\lim c_{n}\left(\phi_{n}\right)$. We claim that $c$ is well defined. Indeed, if $\left(\psi_{n}\right)_{n}$ is another sequence such that $\lim _{n} d_{\mathrm{cb}}\left(\psi_{n}, \phi\right)=0$, then $\lim _{n} d_{\mathrm{cb}}\left(\psi_{n}, \phi_{n}\right)=0$. Using the fact that each $c_{n}$ is 1-Lipschitz, this implies that $\lim _{n}\left|c_{n}\left(\psi_{n}\right)-c_{n}\left(\phi_{n}\right)\right|_{\infty}=0$. Since $\mathcal{U}$ is nonprincipal, we conclude that $\mathcal{U}-\lim c_{n}\left(\phi_{n}\right)=\mathcal{U}-\lim c_{n}\left(\psi_{n}\right)$. Let $\varepsilon>0$ be arbitrary. By the assumption (4') there exists $\alpha \in \operatorname{Aut}^{\mathrm{C}}(E) \operatorname{such}$ that $\operatorname{Osc}\left(c \uparrow\left(\alpha \circ \operatorname{Emb}^{\mathrm{C}}(X, Y)\right) \leqslant \varepsilon\right.$. Let $D \subseteq \operatorname{Emb}_{\delta}^{\mathrm{C}}(X, Y)$ be a finite $\varepsilon$-dense subset. Using the definition of $c$ and the fact that $c$ stabilizes on $\left.\alpha \circ \operatorname{Emb}_{\delta}^{\mathrm{C}}(X, Y)\right)$, choose $n \in \mathbb{N}$ such that:

- for every $\phi \in D$ there exists $\bar{\phi} \in \operatorname{Emb}^{\mathrm{C}}\left(X, Z_{n}\right)$ such that $d_{\mathrm{cb}}(\alpha \circ \phi, \bar{\phi}) \leqslant \varepsilon$, and such that for every $\phi, \psi \in D$ one has that $\left|c_{n}(\bar{\phi})-c_{n}(\bar{\psi})\right| \leqslant \varepsilon$.

- there is $\gamma \in \operatorname{Emb}^{\mathrm{C}}\left(Y, Z_{n}\right)$ such that $d_{\mathrm{cb}}(\alpha \uparrow Y, \gamma) \leqslant \varepsilon$.

It follows that $d_{\mathrm{cb}}(\bar{\phi}, \gamma \circ \phi) \leqslant 2 \varepsilon$ for every $\phi \in D$. Hence, $\left|c_{n}(\gamma \circ \phi)-c_{n}(\gamma \circ \psi)\right| \leqslant 5 \varepsilon+\varpi(\delta)$ for every $\phi, \psi \in D$. Consequently, $c_{n}$ has oscillation at most $7 \varepsilon+\varpi(\delta)$ on $\gamma \circ \operatorname{Emb}_{\delta}^{\mathrm{C}}(X, Y)$. Since $\varepsilon$ is an arbitrary positive real number, this contradicts the assumption that $c_{n}$ is a "bad" coloring witnessing the failure of $\left(7^{\prime}\right)$.

The equivalence of (5) $-(7)$ is done in Proposition 2.12 .

(7) implies (4): Fix $X \subset Y \subset E$ that belong to $\mathcal{A}, \varepsilon>0$, and $r \in \mathbb{N}$. Let $Z \in\langle\mathcal{A}\rangle$ be witnessing that

(7) holds for the given data. Since $E$ is universal for $\langle\mathcal{A}\rangle$, we may assume that $Z$ is a substructure of $E$. Given an $r$-coloring $c$ of $\mathrm{Emb}^{\mathrm{C}}(X, E)$, we can take its restriction to $\operatorname{Emb}^{\mathrm{C}}(X, Z)$, and then find $\gamma \in \operatorname{Emb}^{\mathrm{C}}(Y, Z)$ such that $\gamma \circ \operatorname{Emb}^{\mathrm{C}}(X, Y)$ is $\varepsilon$-monochromatic for $c$. Finally, let $\alpha \in \operatorname{Aut}^{\mathrm{C}}(E)$ be such that $d_{\mathrm{cb}}(\alpha \uparrow Y, \gamma) \leqslant \varepsilon$. It follows that $c 2 \varepsilon$-stabilizes on $\alpha \circ \operatorname{Emb}^{\mathrm{C}}(X, Y)$.

2.3.2. The Dual Ramsey Theorem. The proof of the (ARP) properties of several classes of $R$-operator spaces and systems is based on the Dual Ramsey Theorem (DRT) of R. L. Graham and B. L. Rothschild [22]. Its statement is commonly presented by using partitions, but for practical purposes, we recall it in terms of rigid surjections between finite linear orderings. Given two linear orderings $\left(R,<_{R}\right)$ and $\left(S,<_{S}\right)$, an onto map $f: R \rightarrow S$ is a rigid surjection when $\min _{R} f^{-1}\left(s_{0}\right)<\min _{R} f^{-1}\left(s_{1}\right)$ for every $s_{0}, s_{1} \in S$ such that $s_{0}<_{S} s_{1}$. The class of rigid surjections from $R$ onto $S$ is denoted by $\operatorname{Epi}(R, S)$.

Theorem 2.17 ((DRT) [22]). For every finite linear orderings $R$ and $S$ and every $r \in \mathbb{N}$ there exists an integer $n \in \mathbb{N}$ such that, considering $n$ naturally ordered, every $r$-coloring of $\operatorname{Epi}(n, R)$ has a monochromatic set of the form $\operatorname{Epi}(S, R) \circ \gamma=\{\sigma \circ \gamma: \sigma \in \operatorname{Epi}(S, R)\}$ for some $\gamma \in \operatorname{Epi}(n, S)$.

\section{The RAMSEY PROPERTY FOR OPERATOR SPACES}

The aim of this section is to prove the extreme amenability of the automorphism groups of operator spaces which are Fraïssé limits of certain classes of finite-dimensional injective operator spaces. 
3.1. Fraïssé limits of exact operator spaces. Recall that an operator space $E$ is injective if it is injective in the category of operator spaces; that is, whenever $X \subset Y$ are operator spaces, any completely contractive map $\phi: X \rightarrow E$ can be extended to a completely contractive map $\psi: Y \rightarrow E$. The finite-dimensional injective operator spaces are precisely the ones of the form $M_{q_{1}, s_{1}} \oplus_{\infty} \cdots \oplus_{\infty}$ $M_{q_{n}, s_{n}}$ for $n, q_{1}, s_{1}, \ldots, q_{n}, s_{n} \in \mathbb{N}$. Here, $M_{q, s}$ is the space of $q \times s$ matrices with complex entries, regarded as a space of operators on the $(q+s)$-dimensional Hilbert space of the form

$$
\left[\begin{array}{ll}
0 & * \\
0 & 0
\end{array}\right],
$$

where the diagonal blocks have size $q \times q$ and $s \times s$. The operator spaces $M_{q, 1}$ and $M_{1, q}$ are called the $q$-dimensional column operator Hilbert space and the $q$-dimensional row operator Hilbert space, respectively. The space $M_{q, q}$ of $q \times q$ matrices will be simply denoted by $M_{q}$, and the $n$-fold $\infty$-sum of $M_{q, s}$ by itself will be denoted by $\ell_{\infty}^{n}\left(M_{q, s}\right)$. It is known that the class of finite-dimensional injective operator spaces coincides with the class of finite-dimensional ternary rings of operator; see [57]. The finite-dimensional commutative ternary rings of operators are precisely the spaces $\ell_{\infty}^{n}$ for $n \in \mathbb{N}[6$, Subsection 8.6.4], which are precisely the finite-dimensional injective minimal operator spaces.

Definition 3.1 (Injective classes). We say that a family of finite-dimensional operator spaces is an injective class of operator spaces if it is one of the following families

- $\mathbb{I}_{1}:=\left\{\ell_{\infty}^{n}\right\}_{n \in \mathbb{N}}$,

- $\mathbb{I}_{q}:=\left\{\ell_{\infty}^{n}\left(M_{q}\right)\right\}_{n \in \mathbb{N}}$,

- $\mathbb{I}_{\mathrm{c}}:=\left\{\ell_{\infty}^{n}\left(M_{q, 1}\right)\right\}_{n, q \in \mathbb{N}}$,

- $\mathbb{I}_{\mathrm{e}}:=\left\{M_{q}\right\}_{q \in \mathbb{N}}$, and

- $\mathbb{I}_{\text {inj }}=\left\{M_{q_{1}, s_{1}} \oplus_{\infty} \cdots \oplus_{\infty} M_{q_{n}, s_{n}}\right\}_{n, q_{1}, s_{1}, \ldots, q_{n}, s_{n} \in \mathbb{N}}$.

If $\mathbb{I}$ is any of the classes in Definition 3.1, then $\mathbb{I}$ satisfies the assumptions of Theorem 2.7. Thus, $\mathbb{I}$ is a stable amalgamation class and $\langle\mathbb{I}\rangle$ is a stable Fraïssé class with modulus $\varpi(\delta)=\delta$. The corresponding classes [I] can be described as follows.

Definition 3.2 (Spaces locally approximated by injective classes).

(a) $\left[\mathbb{I}_{1}\right]$ is the class of minimal operator spaces, which can be identified with the class of Banach spaces.

(b) $\left[\mathbb{I}_{q}\right]$ is the class of $q$-minimal operator spaces (see [34]).

(c) $\left[\mathbb{I}_{\mathrm{c}}\right]$ is the class of operator sequence spaces (see [33]).

(d) $\left[\mathbb{I}_{\mathrm{e}}\right]=\left[\mathbb{I}_{\mathrm{inj}}\right]$ is the class of exact operator spaces (see [52], [54, Theorem 17.1]).

Observe that Banach spaces (endowed with their minimal operator space structure) coincide with 1-minimal operator spaces. Their limits are the following.

\section{Example 3.3.}

- FLim $\mathbb{I}_{1}$ is the Gurarij space $\mathbb{G}[4,25,32,39]$.

- FLim $\mathbb{I}_{q}$ is the q-minimal Gurarij space $\mathbb{G}_{q}$ [37, §6.5].

- FLim $\mathbb{I}_{\mathrm{c}}$ is the Gurarij column space $\mathbb{G} \mathbb{C}$ [37, \$6.3].

- FLim $\mathbb{I}_{\mathrm{e}}$ is the noncommutative Gurarij space $\mathbb{N G}$ [37, \$8.1].

Observe that the Gurarij space $\mathbb{G}$ coincides with $\mathbb{G}_{1}$ in the notation of Example 3.3. In fact, the original definition of the Gurarij space considered by Gurarij [25] and Lusky [39, 40, 42] looks somewhat 
different. The original characterization of $\mathbb{G}$ is as the unique separable Banach space satisfying the following extension property: for every finite-dimensional Banach spaces $E \subseteq F$, linear contraction $\phi: E \rightarrow \mathbb{G}$, and $\varepsilon>0$, there exists an extension $\hat{\phi}: F \rightarrow \mathbb{G}$ satisfying $\|\hat{\phi}\|<1+\varepsilon$. The fact that such a definition is equivalent to the one given in Example 3.3 is proved in [4]. Similarly, the original notion of noncommutative Gurarij space considered by Oikhberg in [46] and proved to be unique in $[36]$ is the following: a separable exact operator space $\mathbb{N} \mathbb{G}$ such that for every finite-dimensional exact operator spaces $E \subseteq F$, linear complete contraction $\phi: E \rightarrow \mathbb{N} \mathbb{G}$, and $\varepsilon>0$, there exists an extension $\hat{\phi}: F \rightarrow \mathbb{G}$ satisfying $\|\hat{\phi}\|_{\mathrm{cb}}<1+\varepsilon$. The fact that such a definition is equivalent to the one given in Example 3.3 is established in [36].

3.2. The approximate Ramsey property and extreme amenability. Fix $q, s \in \mathbb{N}$. The goal of this part is to prove that the classes $\mathbb{I}$ of finite-dimensional exact operator spaces introduced above satisfy the stable Ramsey property with modulus $\varpi(\delta)=\delta$. Since $\mathbb{I}$ and its generated class $\langle\mathbb{I}\rangle$ are both stable Fraïssé classes, Proposition 2.12 tell us that it suffices to prove the discrete approximate Ramsey property of each $\mathbb{I}$.

Lemma 3.4 (ARP of $\left.\left\{\ell_{\infty}^{d}\left(M_{q, s}\right)\right\}_{d \in \mathbb{N}}\right)$. Fix $q, s \in \mathbb{N}$. For every $d, m, r \in \mathbb{N}$ and $\varepsilon>0$ there exists $n \in \mathbb{N}$ such that every $r$-coloring of $\operatorname{Emb}\left(\ell_{\infty}^{d}\left(M_{q, s}\right), \ell_{\infty}^{n}\left(M_{q, s}\right)\right)$ has an $\varepsilon$-monochromatic set of the form $\gamma \circ \operatorname{Emb}\left(\ell_{\infty}^{d}\left(M_{q, s}\right), \ell_{\infty}^{m}\left(M_{q, s}\right)\right)$ for some $\gamma \in \operatorname{Emb}\left(\ell_{\infty}^{m}\left(M_{q, s}\right), \ell_{\infty}^{n}\left(M_{q, s}\right)\right)$.

Similarly as it was done for the proof of the (ARP) of the class of Banach spaces $\left\{\ell_{\infty}^{n}\right\}_{n}$ in [3], rather than proving Lemma 3.4 directly, we will establish its natural dual statement, which is Lemma 3.5 below. Given two operator spaces $X$ and $Y$, let $\mathrm{CQ}(X, Y)$ be the set of completely contractive quotient mappings $\phi: X \rightarrow Y$, i.e. $\phi: X \rightarrow Y$ such that each amplification $\phi^{(n)}$ is a contractive quotient mapping $[13, \S 2.2]$. Notice that this is equivalent to the assertion that the dual map $\phi^{*}: Y^{*} \rightarrow X^{*}$ is a completely isometric embedding. We denote by $T_{s, q}$ the operator space dual of $M_{q, s}$. This can be identified as the space of $s \times q$ matrices with matrix norms given by the normalized Hilbert-Schmidt norm $[13, \S 1.2]$. The duality between $M_{q, s}$ and $T_{s, q}$ is implemented by the paring $(\alpha, \beta) \mapsto \operatorname{Tr}(\alpha \beta)$, there $\operatorname{Tr}$ denotes the normalized trace for $s \times s$ matrices. One can then canonically identify the operator space dual of $\ell_{\infty}^{d}\left(M_{q, s}\right)$ with the 1 -sum $\ell_{1}^{d}\left(T_{s, q}\right)$ of $d$ copies of $T_{s, q}$. From this it is easy to see that Lemma 3.4 and Lemma 3.5 below are equivalent by duality.

Lemma 3.5. Fix $q, s \in \mathbb{N}$. For every $d, m, r \in \mathbb{N}$ and $\varepsilon>0$ there exists $n \in \mathbb{N}$ such that every $r$-coloring of $\mathrm{CQ}\left(\ell_{1}^{n}\left(T_{s, q}\right), \ell_{1}^{d}\left(T_{s, q}\right)\right)$ has an $\varepsilon$-monochromatic set of the form $\mathrm{CQ}\left(\ell_{1}^{m}\left(T_{s, q}\right), \ell_{1}^{d}\left(T_{s, q}\right)\right) \circ \gamma$ for some $\gamma \in \mathrm{CQ}\left(\ell_{1}^{n}\left(T_{s, q}\right), \ell_{1}^{m}\left(T_{s, q}\right)\right)$.

In order to prove Lemma 3.5 we will need the following fact about linear complete isometries, which is an immediate consequence of [36, Lemma 5.17]; see also [10, Lemma 3.6].

Lemma 3.6. Suppose that $q, s, q_{1}, s_{1}, \ldots, q_{n}, s_{n} \in \mathbb{N}$, and $\phi_{i}: M_{q, s} \rightarrow M_{q_{i}, s_{i}}$ are completely contractive linear maps for $i=1,2, \ldots, n$. Then the linear map $\phi: M_{q, s} \rightarrow M_{q_{1}, s_{1}} \oplus_{\infty} \cdots \oplus_{\infty} M_{q_{n}, s_{n}}, x \mapsto$ $\left(\phi_{1}(x), \ldots, \phi_{n}(x)\right)$ is a complete isometry if and only if $\phi_{i}$ is a complete isometry for some $i \leqslant n$.

The proof of Lemma 3.5 relies on the Dual Ramsey Theorem 2.17 by Graham and Rothschild.

Proof of Lemma 3.5. Fix $d, m, r \in \mathbb{N}$ and $\varepsilon>0$. We identify a linear map $\phi$ from $\ell_{1}^{n}\left(T_{s, q}\right)$ to $\ell_{1}^{d}\left(T_{s, q}\right)$ with a $d \times n$ matrix $\left[\phi_{i j}\right]$ where $\phi_{i j}: T_{s, q} \rightarrow T_{s, q}$ is a linear map. It follows from (the dual of) Lemma 3.6 that $\phi$ is a completely contractive quotient mapping if and only if every row of $\left[\phi_{i j}\right]$ has an entry 
that is a surjective complete isometry of $T_{s, q}$, and every column is a complete contraction from $T_{s, q}$ to $\ell_{1}^{d}\left(T_{s, q}\right)$. This implies that if a column has an entry that is a surjective complete isometry of $T_{s, q}$, then all the other entries of the column are zero. Let now $\mathcal{P}$ be a finite set of complete contractions from $T_{s, q}$ to $\ell_{1}^{d}\left(T_{s, q}\right)$ —which we regard as $d$-dimensional column vectors with entries from $T_{s, q}$-with the following properties:

(i) the zero map belongs to $\mathcal{P}$;

(ii) for every $i \leqslant d$ the canonical embedding of $T_{s, q}$ into the $i$-th coordinate of $\ell_{1}^{d}\left(T_{s, q}\right)$ belongs to $\mathcal{P}$;

(iii) for every nonzero complete contraction $\phi: T_{s, q} \rightarrow \ell_{1}^{d}\left(T_{s, q}\right)$ there exists a nonzero element $\phi_{0}$ of $\mathcal{P}$ such that $\left\|\phi-\phi_{0}\right\|_{\mathrm{cb}}<\varepsilon$ and $\left\|\phi_{0}\right\|_{\mathrm{cb}}<\|\phi\|_{\mathrm{cb}}$.

Fix $\varepsilon_{0}>0$ small enough and a finite $\varepsilon_{0}$-dense subset $U$ of the group of automorphisms of $T_{s, q}$. Let $\mathcal{Q}$ be the (finite) set of linear complete isometries from $\ell_{1}^{d}\left(T_{s, q}\right)$ to $\ell_{1}^{m}\left(T_{s, q}\right)$ such that every row contains at most one nonzero entry, every column exactly one nonzero entry, and every nonzero entry is an automorphism of $T_{s, q}$ that belongs to $U$. Fix any linear order $<$ on $\mathcal{Q}$, and a linear order on $\mathcal{P}$ with the property that

$$
\phi<\phi^{\prime} \text { whenever }\|\phi\|_{\mathrm{cb}}<\left\|\phi^{\prime}\right\|_{\mathrm{cb}} .
$$

Endow $\mathcal{Q} \times \mathcal{P}$ with the corresponding antilexicographic order. An element of $\operatorname{Epi}(n, \mathcal{P})$ is an $n$-tuple $\bar{v}=$ $\left(v_{1}, \ldots, v_{n}\right)$ of elements of $\mathcal{P}$. We associate with such an $n$-tuple the element $\alpha_{\bar{v}}$ of $\operatorname{CQ}\left(\ell_{1}^{n}\left(T_{s, q}\right), \ell_{1}^{d}\left(T_{s, q}\right)\right)$ whose representative matrix has $v_{i}$ as $i$-th column for $i=1,2, \ldots, n$. Similarly an element of $\operatorname{Epi}(n, \mathcal{Q} \times$ $\mathcal{P})$ is an $n$-tuple $(\bar{B}, \bar{w})=\left(B_{1}, w_{1}, \ldots, B_{n}, w_{n}\right)$. We associate with such an $n$-tuple the element $\alpha_{(\bar{B}, \bar{w})}$ of $\operatorname{CQ}\left(\ell_{1}^{n}\left(T_{s, q}\right), \ell_{1}^{m}\left(T_{s, q}\right)\right)$ with $B_{i} w_{i}$ as $i$-th column for $i=1,2, \ldots, n$. Suppose now that $n \in \mathbb{N}$ is obtained from $\mathcal{P}$ and $\mathcal{Q} \times \mathcal{P}$ by applying the dual Ramsey theorem, Theorem 2.17. We claim that such an $n$ satisfies the desired conclusions. Suppose that $c$ is an $r$-coloring of $\mathrm{CQ}\left(\ell_{1}^{n}\left(T_{s, q}\right), \ell_{1}^{d}\left(T_{s, q}\right)\right)$. The map $\bar{v} \mapsto \alpha_{\bar{v}}$ from $\operatorname{Epi}(n, \mathcal{P})$ to $\mathrm{CQ}\left(\ell_{1}^{n}\left(T_{s, q}\right), \ell_{1}^{d}\left(T_{s, q}\right)\right)$ induces an $r$-coloring on $\operatorname{Epi}(n, \mathcal{P})$. By the choice of $n$ there exists an element $(\bar{B}, \bar{w})$ of $\operatorname{Epi}(n, \mathcal{Q} \times \mathcal{P})$ such that any rigid surjection from $n$ to $\mathcal{P}$ that factors through $(\bar{B}, \bar{w})$ has a fixed color $i \in r$. To conclude the proof it remains to show the following.

Claim 3.6.1. The set of completely contractive quotient mappings from $\ell_{1}^{n}\left(T_{s, q}\right)$ to $\ell_{1}^{d}\left(T_{s, q}\right)$ that factor through $\alpha_{(\bar{B}, \bar{w})}$ is $\varepsilon$-monochromatic.

Proof of Claim: This will follow once we show that, given any $\rho \in \operatorname{CQ}\left(\ell_{1}^{m}\left(T_{s, q}\right), \ell_{1}^{d}\left(T_{s, q}\right)\right)$ there exists $\tau \in \operatorname{Epi}(\mathcal{Q} \times \mathcal{P}, \mathcal{P})$ such that $\left\|\alpha_{\tau(\bar{B}, \bar{w})}-\rho \circ \alpha_{(\bar{B}, \bar{w})}\right\|_{\mathrm{cb}} \leqslant \varepsilon$, where we denoted by $\tau(\bar{B}, \bar{w})$ the element $\left(\tau\left(B_{1}, w_{1}\right), \ldots, \tau\left(B_{n}, w_{n}\right)\right)$ of $\operatorname{Epi}(n, \mathcal{P})$. If $\rho$ has representative matrix $A$, then this is equivalent to the assertion that, for every $i \leqslant n, \tau\left(B_{i}, w_{i}\right)$ has cb-distance at most $\varepsilon$ from $A B_{i} w_{i}$. We proceed to define such a rigid surjection $\tau$ from $\mathcal{Q} \times \mathcal{P}$ to $\mathcal{P}$. By the structure of completely contractive quotient mappings from $\ell_{1}^{m}\left(T_{s, q}\right)$ to $\ell_{1}^{d}\left(T_{s, q}\right)$ recalled above, there exists $A^{\dagger} \in \mathcal{Q}$ such that $\left\|A A^{\dagger}-\operatorname{Id}_{\ell_{1}^{d}\left(T_{s, q}\right)}\right\|_{\mathrm{cb}} \leqslant \varepsilon$, provided that $\varepsilon_{0}$ is small enough (depending only from $\varepsilon$ ). Define now $\tau: \mathcal{Q} \times \mathcal{P} \rightarrow \mathcal{P}$ by letting, for $B \in \mathcal{Q}$ and $w \in \mathcal{P}, \tau(B, w)=0$ if $w=0, \tau(B, w)=w$ if $B=A^{\dagger}$, and otherwise $\tau(B, w) \in \mathcal{P}$ such that $0<\|\tau(B, w)\|_{\mathrm{cb}}<\|A B w\|_{\mathrm{cb}}$ and $\|\tau(B, w)-A B w\|_{\mathrm{cb}}<\varepsilon$. It is clear from the definition that $\tau(B, w)$ has distance at most $\varepsilon$ from $A B w$. We need to verify that $\tau$ is indeed a rigid surjection from $\mathcal{Q} \times \mathcal{P}$ to $\mathcal{P}$. Observe that $\tau$ is onto, and the pairs $(B, 0)$ are the only elements of $\mathcal{Q} \times \mathcal{P}$ that are mapped by $\tau$ to zero. It is therefore enough to prove that, for every $w \in \mathcal{P},\left(A^{\dagger}, w\right)$ is the minimum of the preimage of $w$ under $\tau$. Suppose that $\left(B^{\prime}, w^{\prime}\right)$ is an element of the preimage of $w$ under $\tau$. Then by definition 
of $\tau$ we have that

$$
\|w\|_{\mathrm{cb}}<\left\|A B w^{\prime}\right\|_{\mathrm{cb}} \leqslant\left\|w^{\prime}\right\|_{\mathrm{cb}} .
$$

By our assumptions on the ordering of $\mathcal{P}$, it follows that $w<w^{\prime}$ and hence $\left(A^{\dagger}, w\right)<\left(B^{\prime}, w^{\prime}\right)$. This concludes the proof.

Using the general facts about the approximate Ramsey property from Proposition 2.12, one can bootstrap the approximate Ramsey property from the class considered in Lemma 3.5 to other classes of operator spaces. In fact one can obtain the compact stable Ramsey property with modulus $\varpi(\delta)=\delta$; see Definition 2.9.

Theorem 3.7. The following classes of finite-dimensional operator spaces satisfy the compact (SRP) with modulus $\varpi(\delta)=\delta$ :

(1) for every $q \in \mathbb{N}$, the injective class $\mathbb{I}_{q}$ and the class $\left\langle\mathbb{I}_{q}\right\rangle$ of finite-dimensional q-minimal operator spaces, and in particular the class of finite-dimensional Banach spaces.

(2) The injective class $\mathbb{I}_{\mathrm{c}}$ and the class $\left\langle\mathbb{I}_{\mathrm{c}}\right\rangle$ of finite-dimensional operator sequence spaces.

(3) The classes $\mathbb{I}_{\mathrm{e}}, \mathbb{I}_{\mathrm{inj}}$ and the class $\left\langle\mathbb{I}_{\mathrm{e}}\right\rangle=\left\langle\mathbb{I}_{\mathrm{inj}}\right\rangle$ of finite-dimensional exact operator spaces.

Proof. In each of the cases, it is enough to verify that the given class satisfies the discrete (ARP) in view of Proposition 2.10 and the equivalence of (1) and (5) in Proposition 2.12.

(1): It follows from Lemma 3.4 for $q=r$ that $\mathbb{I}_{q}$ satisfies the discrete (ARP).

(2): Lemma 3.4 for $s=1$ is the discrete (ARP) of $\mathbb{I}_{\mathrm{c}}$.

(3): We verify that the class of finite dimensional exact operator spaces $\left\langle\mathbb{I}_{e}\right\rangle$ satisfies the (ARP). By the equivalence of (1) and (6) in Proposition 2.12, it suffices to show that for every positive integer $p, q, r$ such that $p \leqslant q$, and every $\varepsilon>0$ there is some finite-dimensional exact operator space $Z$ such that every $r$-coloring of $\operatorname{Emb}\left(M_{p}, Z\right) \varepsilon$-stabilizes on $\gamma \circ \operatorname{Emb}\left(M_{p}, M_{q}\right)$ for some $\gamma \in \operatorname{Emb}\left(M_{q}, Z\right)$. Now, $M_{p}$ and $M_{q}$ are $q$-minimal, so by (1) there is such a $Z$ which is a finite-dimensional $q$-minimal operator space. Since every $q$-minimal operator space is exact, this concludes that $\left\langle\mathbb{I}_{\mathrm{e}}\right\rangle$ satisfies the (ARP). It follows from the equivalence (1) and (5) in Proposition 2.12 that both $\mathbb{I}_{e}$ and $\mathbb{I}_{\text {inj }}$ also satisfies the (ARP).

Theorem 3.8 below extends the work on the Gurarij space from [3, Theorem 2.5]. It is a consequence of the KPT correspondence as in Proposition 2.16 and corresponding approximate Ramsey properties in Theorem 3.7. It shows that the limits of the Fraïssé classes mentioned in Definition 3.3 have extremely amenable automorphism groups.

Theorem 3.8. The following operator spaces have extremely amenable automorphism groups:

(1) each q-minimal Gurarij space $\mathbb{G}_{q}$, and in particular the Gurarij space $\mathbb{G}$.

(2) The column Gurarij space $\mathbb{C} \mathbb{G}$.

(3) The noncommutative Gurarij space $\mathbb{N} \mathbb{G}$.

Remark 3.9. One can also give a direct, quantitative proof of the ARP for finite-dimensional Banach spaces using Lemma 3.5 when $s=q=1$ and the injective envelope construction [6, Subsection 4.2]. Such a proof yields an explicit bound of the Ramsey numbers for the class of finite-dimensional Banach spaces in terms of the Ramsey numbers for the Dual Ramsey Theorem. Furthermore the same argument applies with no change in the case of real Banach spaces, yielding extreme amenability of the group of surjective linear isometries of the real Gurarij space (see [2, Appendix A]). 


\subsection{Operator spaces with a distinguished functional.}

3.3.1. Fraïssé limits of $R$-operator spaces. The natural geometric object associated with a Banach space $X$ is the unit ball $\operatorname{Ball}\left(X^{*}\right)$ of the dual of $X$, i.e. the $w^{*}$-compact convex symmetric set of contractive linear functionals on $X$. As discussed in the introduction, the noncommutative analog of such a correspondence involves the notion of matrix functionals. Given an operator space $X$, a matrix functional on $X$ is a linear function from $X$ to $M_{q, r}(\mathbb{C})$ for some $q, r \in \mathbb{N}$. The space $\operatorname{CBall}\left(X^{*}\right)$ is the sequence $\left(K_{q, r}\right)_{q, r \in \mathbb{N}}$, where $K_{q, r}$ is the $w^{*}$-compact convex set of completely contractive matrix functionals from $X$ to $M_{q, r}(\mathbb{C})$. The space $\mathrm{CBall}\left(X^{*}\right)$ is endowed with a notion of rectangular matrix convex combinations that makes it a compact rectangular matrix convex set [19]. Furthermore any compact rectangular convex set arises in this way. It should be clear from this that matrix functionals provide the right noncommutative analog of functionals on Banach spaces.

More generally, suppose that $R$ is a separable nuclear operator space, that is, the identity map of $R$ is the pointwise limit of completely contractive maps that factor through finite-dimensional injective operator spaces. When $R$ is in addition a minimal operator space (i.e. a Banach space), this is equivalent to the assertion that $R$ is a Lindenstrauss Banach space [6, Subsection 8.6.4]. A classical result of Wojtaszczyk [59] asserts that the separable Lindenstrauss spaces are precisely the separable Banach spaces that are isometric to the range of a contractive projection on the Gurarij space $\mathbb{G}$. The noncommutative analog of such a result asserts that the separable nuclear operator spaces are precisely the separable operator spaces that are completely isometric to the range of a completely contractive projection on the noncommutative Gurarij space [35]. A similar result holds for operator sequence spaces in terms of the column Gurarij space [37, Subsection 6.5]. Notice that injective finite-dimensional operator spaces are always nuclear, but the converse does not hold. The following result can be found in $[37, \S 6.5, \S 6.6]$.

Proposition 3.10. Let $R$ be a separable operator space.

(1) Suppose that $R$ is q-minimal. Then $R$ is nuclear if and only if it is $\mathbb{I}_{q}$-nuclear, i.e. the identity on $R$ is the pointwise limit of completely contractive maps that factor through some space in $\mathbb{I}_{q}$. In particular, nuclear q-minimal separable operator spaces belong to $\left[\mathbb{I}_{q}\right]$.

(2) Suppose that $R$ is an operator sequence space. Then $R$ is nuclear if and only it is $\mathbb{I}_{\mathrm{c}}-$ nuclear, i.e. the identity on $R$ is the pointwise limit of completely contractive maps that factor through some space in $\mathbb{I}_{\mathrm{c}}$. In particular, nuclear separable sequence operator spaces belong to $\left[\mathbb{I}_{c}\right]$.

Definition 3.11 ( $R$-functionals). For an operator space $X$ and a separable nuclear operator space $R$, an $R$-functional on $X$ is a completely bounded linear operator from $X$ to $R$. Let $\operatorname{CC}(X, R)$ be the space of completely contractive $R$-functionals on $X$, regarded as a Polish space with the topology of pointwise convergence. Let $\operatorname{Aut}(X) \curvearrowright \mathrm{CC}(X, R)$ be the continuous action $(\alpha, s) \mapsto s \circ \alpha^{-1}$. Finally, given $s \in \mathrm{CC}(X, R)$, let $\operatorname{Aut}(X, s) \subseteq \operatorname{Aut}(X)$ be the stabilizer of $s$ with respect to such an action.

Recall that given a family $\mathcal{A}$ of operator spaces and a separable operator space $R$, let $\mathcal{A}^{R}$ be the collection of $R$-operator spaces $\boldsymbol{X}=\left(X, s_{X}\right)$ where $X \in \mathcal{A}$ and $s_{X}: X \rightarrow R$ is a complete contraction.

Proposition 3.12. Let II be an injective class of operator spaces as in Definition 3.1, and let $R$ be a separable operator space. Then

(1) $\mathbb{I}^{R}$ is a stable amalgamation class with stability modulus $\varpi(\delta)=2 \delta$ when $R \in \mathbb{I}$.

(2) $\langle\mathbb{I}\rangle^{R}$ is a stable Fraïssé class with stability modulus $\varpi(\delta)=2 \delta$. 
(3) Suppose that in addition $R$ is nuclear. Then the Fraïssé limit of $\langle\mathbb{I}\rangle^{R}$ is the $R$-operator space $\left(\mathrm{FLim} \mathbb{I}, \Omega_{\mathrm{FLim} \mathbb{I}}^{R}\right)$.

Proof. This is a consequence of Proposition 2.8. Obviously both $\mathbb{I}^{R}$ and $\langle\mathbb{I}\rangle^{R}$ have a minimal element, so it suffices to show that these classes satisfy the (SAP) with modulus $2 \delta$. Every finite-dimensional subspace of $R$ embeds into an element of $\langle\mathbb{I}\rangle$. Consequently, (1) and (2) immediately follow from Proposition 2.8 (1). (3) is also consequence of Proposition 2.8 and the fact that FLim $\mathbb{I}=$ FLim $\langle\mathbb{I}\rangle$.

The $R$-functional $\Omega_{\mathrm{FLim} I}^{R}$ as in Proposition 3.12 is called the generic completely contractive $R$ functional on FLim II. The name is justified by the fact that the Aut(FLim $\mathbb{I}$ )-orbit of $\Omega_{\mathrm{FLim} \mathbb{I}}^{R}$ is a dense $G_{\delta}$ subset of the space CC(FLim $\left.\mathbb{I}, R\right)$ of completely contractive $R$-functionals on FLim $\mathbb{I}$.

3.3.2. KPT correspondence and the approximate Ramsey property of $R$-operator spaces. We present the approximate Ramsey properties of several classes of $R$-operator spaces, and the corresponding extreme amenability of the automorphism group of their Fraïssé limits.

Theorem 3.13. The following classes of finite-dimensional $R$-operator spaces satisfy the compact (SRP) with stability modulus $\varpi(\delta)=2 \delta$ :

(1) for a q-minimal separable nuclear operator space $R$, the class $\mathbb{I}_{q}^{R}$, if $R$ is finite dimensional, and the class $\left\langle\mathbb{I}_{q}\right\rangle^{R}$ of finite-dimensional q-minimal $R$-operator spaces; in particular, for a separable Lindenstrauss space $R$, the class of $R$-Banach spaces (which recovers [3, Theorem 2.41]).

(2) For a separable nuclear operator sequence space $R$, the class $\mathbb{I}_{\mathrm{c}}^{R}$, if $R$ is finite dimensional, and the class $\left\langle\mathbb{I}_{\mathrm{c}}\right\rangle^{R}$ of finite-dimensional $R$-operator sequence spaces.

(3) For a separable nuclear operator space $R$, the class $\mathbb{I}_{\mathrm{e}}^{R}$, if $R$ is finite dimensional, and the class $\left\langle\mathbb{I}_{\mathrm{e}}\right\rangle^{R}$ of finite-dimensional exact $R$-operator spaces.

Proof. We have seen in Theorem 3.7 that the injective classes $\mathbb{I}_{q}, \mathbb{I}_{c}, \mathbb{I}_{\mathrm{e}}$ and $\mathbb{I}_{\text {inj }}$, and the corresponding completions $\left\langle\mathbb{I}_{q}\right\rangle,\left\langle\mathbb{I}_{c}\right\rangle$ and $\left\langle\mathbb{I}_{\mathrm{e}}\right\rangle=\left\langle\mathbb{I}_{\text {inj }}\right\rangle$ satisfy the compact (SRP) with modulus $\delta$, so it follows from Proposition 2.13 (1) that the corresponding $R$-classes also satisfy the compact (SRP) with modulus $\varpi(\delta)=2 \delta$ (here we are using that for classes satisfying the (SAP) with that modulus, the compact (SRP) and the (SRP) are equivalent).

From Theorem 3.13 and the characterization of extreme amenability in Proposition 2.16 we obtain new extremely amenable groups, extending the work done in [3, Theorem 2.39] for the Gurarij space, and the trivial space $R=\{0\}$ done above in Theorem 3.8.

Corollary 3.14. The following Polish groups are extremely amenable:

(1) The stabilizer of the generic contractive R-functional on the Gurarij space for any separable Lindenstrauss Banach space $R$.

(2) The stabilizer of the generic completely contractive $R$-functional on the q-minimal Gurarij space for any separable q-minimal nuclear operator space $R$.

(3) The stabilizer of the generic completely contractive R-functional on the column Gurarij space for any separable nuclear operator sequence $R$.

(4) The stabilizer of the generic completely contractive $R$-functional on the noncommutative Gurarij space for any separable nuclear operator space $R$.

Again, the same proof shows that (1) of Corollary 3.14 also holds when one considers the real Gurarij space and any real separable Lindenstrauss space $R$. 


\section{The Ramsey property of noncommutative Choquet simplices and operator systems}

In this section we establish the approximate (dual) Ramsey property for noncommutative Choquet simplices with a distinguished point. We will then apply this to compute the universal minimal flows of the automorphisms group of the noncommutative Poulsen simplex. This will be done by studying operator systems with a distinguished ucp map to a fixed nuclear separable operator system $R(R$ operator systems).

4.1. Choquet simplices and operator systems. The correspondence between compact convex sets and function systems admits a natural noncommutative generalization. A compact matrix convex set is a sequence $\boldsymbol{K}=\left(K_{n}\right)$ of sets $K_{n} \subset M_{n}(V)$ for some topological vector space $V$ that is matrix convex [58, Definition 1.1]. This means that whenever $\alpha_{i} \in M_{q_{i}, q}$ and $v_{i} \in K_{q_{i}}$ are such that $\alpha_{1}^{*} \alpha_{1}+\cdots+\alpha_{q}^{*} \alpha_{q}=$ 1 , then the matrix convex combination $\alpha_{1}^{*} v_{1} \alpha_{1}+\cdots+\alpha_{q}^{*} v_{q} \alpha_{q}$ belongs to $K_{q}$. A continuous matrix affine function $\phi: K \rightarrow \boldsymbol{T}$ between compact matrix convex sets is a sequence of continuous functions $\phi_{n}: K_{n} \rightarrow T_{n}$ that is matrix affine in the sense that it preserves matrix convex combinations. The group $\operatorname{Aut}(\boldsymbol{K})$ of matrix affine homeomorphisms of $\boldsymbol{K}$ is a Polish group when endowed with the compact-open topology.

To each operator system $X$ one can canonically assign a compact matrix convex set: the matrix state space $\boldsymbol{S}(X)$. This is the sequence $\left(S_{n}(X)\right)$, where $S_{n}(X) \subset M_{n}\left(X^{*}\right)$ is the space of all ucp maps from $X$ to $M_{n}$. Conversely, to a compact convex set $\boldsymbol{K}$ one can associate an operator system $A(\boldsymbol{K})$ of matrix-affine functions from $\boldsymbol{K}$ to $\mathbb{R}$. It is proved in [58, Section 3] that these constructions are the inverse of each other, and define an equivalence between the category of operator systems and ucp maps, and the category of compact matrix convex sets and continuous matrix affine functions. In particular if $X$ is an operator system, then the group $\operatorname{Aut}(X)$ of surjective unital complete isometries on $X$ can be identified with the group $\operatorname{Aut}(\boldsymbol{K})$ of matrix affine homeomorphisms of the matrix state space $\boldsymbol{K}$ of $X$. The notions of matrix extreme point and matrix extreme boundary can be defined in the setting of compact matrix convex sets by using matrix convex combinations [58].

Recall that an operator system $X$ is called nuclear if its identity map is the pointwise limit of ucp maps that factor through finite-dimensional injective operator systems. When $X=A(K)$, this is equivalent to the assertion that the state space $K$ of $X$ is a Choquet simplex. The matrix state spaces of nuclear operator systems can be seen as the noncommutative generalization of Choquet simplices. The natural noncommutative analog of the Poulsen simplex is studied in [37], where it is proved that finite-dimensional exact operator systems form a Fraïssé class. The matrix state space $\mathbb{N P}=\left(\mathbb{N P}_{n}\right)$ of the corresponding Fraïssé limit $A(\mathbb{N P})$ is a nontrivial noncommutative Choquet simplex with dense matrix extreme boundary, which is called the noncommutative Poulsen simplex in [37, 38].

One can also define a sequence of structures $\left(\mathbb{P}^{(q)}\right)$ for $q \in \mathbb{N}$ that interpolates between the Poulsen simplex and the noncommutative Poulsen simplex, in the context of $q$-minimal operator systems. An operator system is $q$-minimal if it admits a complete order embedding into unital $\mathrm{C}^{*}$-algebra $C\left(K, M_{q}\right)$ for some compact Hausdorff space $K$ [60]. Here we regard the unital selfadjoint subspaces of $C\left(K, M_{q}\right)$ as operator systems, called $q$-minimal operator systems or $M_{q}$-systems. For $q=1$, these are precisely the function systems. A $q$-minimal operator system $X$ can be completely recovered from the portion of the matrix state space only consisting of $S_{k}(X)$ for $k=1,2, \ldots, q$. Conversely a sequence $\left(K_{1}, \ldots, K_{q}\right)$ of compact convex sets $K_{j} \subset M_{j}(V)$ closed under matrix convex combinations $\alpha_{1}^{*} v_{1} \alpha_{1}+\cdots+\alpha_{n}^{*} v_{n} \alpha_{n}$ for $\alpha_{i} \in M_{q_{i} q}$ and $v_{i} \in M_{q_{i}}$ and $q_{i} \leqslant q$ such that $\alpha_{1}^{*} \alpha_{1}+\cdots+\alpha_{n}^{*} \alpha_{n}=1$, uniquely determines a 
$q$-minimal operator system $A\left(K_{1}, \ldots, K_{q}\right)$. The finite-dimensional $q$-minimal operator systems form a Fraïssé class [37, Section 6.7]. The matrix state space $\mathbb{P}^{(q)}=\left(\mathbb{P}_{1}^{(q)}, \ldots, \mathbb{P}_{q}^{(q)}\right)$ of the corresponding limit $A\left(\mathbb{P}^{(q)}\right)$ is the $q$-minimal Poulsen simplex. The model-theoretic properties of $A(\mathbb{P}), A(\mathbb{N P})$, and $A\left(\mathbb{P}^{(q)}\right)$ have been studied in $[20]$.

As we mentioned in the Subsection 2.1, we regard operator systems as objects of the category Osy which has ucp maps as morphisms. The finite-dimensional injective objects in this category are precisely the finite $\infty$-sums of copies of $M_{q}$, which are also the finite-dimensional $\mathrm{C}^{*}$-algebras. The notion of isomorphism in this category coincides with complete order isomorphism. The GromovHausdorff pseudometric of two finite-dimensional operator systems $X, Y$ is the infimum of $\varepsilon>0$ such that there exist ucp maps $f: X \rightarrow Y$ and $g: Y \rightarrow X$ such that $\left\|g \circ f-\operatorname{Id}_{X}\right\|_{\mathrm{cb}}<\varepsilon$ and $\left\|f \circ g-\operatorname{Id}_{Y}\right\|_{\mathrm{cb}}<\varepsilon$. If $X$ and $Y$ are operator systems, then $\operatorname{UCP}(X, Y)$ is the space of ucp maps from $X$ to $Y$, and the automorphism group $\operatorname{Aut}(X)$ is the group of surjective unital complete isometries from $X$ to itself. Both $\operatorname{Aut}(X)$ and $\operatorname{UCP}(X, Y)$ are Polish spaces when endowed with the topology of pointwise convergence. There is a natural continuous action of $\operatorname{Aut}(X)$ on $\operatorname{UCP}(X, Y)$ defined by $(\alpha, s) \mapsto s \circ \alpha^{-1}$. In particular when $Y=M_{q}$ we have that $\operatorname{UCP}(X, Y)=S_{q}(X)$.

Recall that given a class of operator systems $\mathcal{A}$, we denote by $[\mathcal{A}]$ the collection of operator systems $E$ such that every finite-dimensional operator system $X \subseteq E$ is a limit (with respect to the Gromov-Hausdorff pseudometric) of subspaces of operators systems in $\mathcal{A}$, and by $\langle\mathcal{A}\rangle$ the class of finite-dimensional operator systems in $[\mathcal{A}]$.

Definition 4.1 (Injective classes). We say that a family of finite-dimensional operator systems is an injective class of operator systems if it is one of the families $\left\{\ell_{\infty}^{n}\right\}_{n \in \mathbb{N}},\left\{\ell_{\infty}^{n}\left(M_{q}\right)\right\}_{n \in \mathbb{N}}$, or $\left\{M_{q}\right\}_{q \in \mathbb{N}}$.

As mentioned in Theorem 2.7, all the classes of operator systems considered in Definition 4.1 are stable Fraïssé classes with modulus $\varpi(\delta)=2 \delta$. The corresponding generating classes are well-known.

Definition 4.2 (Spaces locally approximated by injective classes).

(a) $\left[\left\{\ell_{\infty}^{n}\right\}_{n \in \mathbb{N}}\right]$ is the class of function systems.

(b) $\left[\left\{\ell_{\infty}^{n}\left(M_{q}\right)\right\}_{n \in \mathbb{N}}\right]$ is the class of q-minimal operator systems (see [60]).

(c) $\left[\left\{M_{q}\right\}_{q \in \mathbb{N}}\right]$ is the class of exact operator systems (see [29]).

And the corresponding limits are the following.

\section{Example 4.3.}

- $\operatorname{FLim}\left\{\ell_{\infty}^{n}\right\}_{n \in \mathbb{N}}$ is the function system $A(\mathbb{P})$ associated with the Poulsen simplex $\mathbb{P}$ (see [37, Section 6.3]).

- $\operatorname{FLim}\left\{M_{q}\right\}_{q \in \mathbb{N}}$ is the operator system $A(\mathbb{N P})$ associated with the noncommutative Poulsen simplex $\mathbb{N P}$ (see [37, Section 8.2]).

- $\operatorname{FLim}\left\{\ell_{\infty}^{n}\left(M_{q}\right)\right\}_{n \in \mathbb{N}}$ is the operator system $A\left(\mathbb{P}^{(q)}\right)$ associated with the q-minimal Poulsen simplex $\mathbb{P}^{(q)}$ (see [37, Section 6.7]).

The main goal of this section is to compute the universal minimal flow of the group Aut $(\mathbb{N P})$ of matrix affine homeomorphisms of the noncommutative Poulsen simplex $\mathbb{N P}$, extending the work in [3, Theorem 3.10] for its commutative version, the Poulsen simplex $\mathbb{P}$. Precisely, we will prove that the universal minimal compact Aut $(\mathbb{N P})$-space is the canonical action of $\operatorname{Aut}(\mathbb{N P})$ on the space $\mathbb{N P}_{1}$ of (scalar) states on $A(\mathbb{N P})$. 
Similarly as in the case of Banach spaces and operator spaces (Section 3.3), we need to consider operator systems with a distinguished (matrix) state. Suppose that $X$ is an operator system. Recall that a state on $X$ is a ucp map from $X$ to $\mathbb{C}$. More generally, an $M_{n}$-state is a ucp map from $X$ to $M_{n}$. Even more generally, if $R$ is any separable nuclear operator system, we call a ucp map from $X$ to $R$ an $R$-state on $X$. As observed above, the space $\operatorname{UCP}(X, R)$ of $R$-states on $X$ is a Polish space endowed with a canonical continuous action of $\operatorname{Aut}(X)$. An $R$-operator system is a pair $\mathbf{X}=\left(X, s_{X}\right)$ of a operator system $X$ and an $R$-state $s_{X}$ on $X$. In the following, we regard $\mathrm{UCP}(X, R)$ as an $\operatorname{Aut}(X)$ space with respect to the canonical action $\operatorname{Aut}(X) \curvearrowright \operatorname{UCP}(X, R)$ given by $(\alpha, s) \mapsto s \circ \alpha^{-1}$. We let $\operatorname{Aut}\left(X, s_{X}\right)$ be the stabilizer of $s_{X} \in \operatorname{UCP}(X, R)$ in $\operatorname{Aut}(X)$. Given a family $\mathcal{A}$ of operator systems, let $\mathcal{A}^{R}$ be the collection of $R$-operator spaces $\left(X, s_{X}\right)$ where $X \in \mathcal{A}$.

Proposition 4.4. Let II be an injective class of operator systems as in Definition 4.1, and suppose that $R$ is a separable operator system such that $R \in[\mathbb{I}]$.

(1) $\mathbb{I}^{R}$ is a stable amalgamation class with stability modulus $\varpi(\delta)=3 \delta$.

(2) $\langle\mathbb{I}\rangle^{R}$ is a stable Fraïssé class with stability modulus $\varpi(\delta)=3 \delta$.

(3) Suppose in addition that $R$ is nuclear. Then the Fraissé limit of $\langle\mathbb{I}\rangle^{R}$ is the $R$-operator system $\left(\mathrm{FLim} \mathbb{I}, \Omega_{\mathrm{FLim} \mathbb{I}}^{R}\right)$.

As in the case of operator spaces, the $R$-state $\Omega_{\text {FLim I }}^{R}$ as in Proposition 4.4 is called the generic $R$-state on FLim II. This is the unique $R$-state on $\Omega_{\mathrm{FLimI}}^{R}$ whose Aut(FLim I)-orbit is a dense $G_{\delta}$ subset of the space $\mathrm{UCP}(\mathrm{FLim} \mathbb{I}, R)$.

4.2. Approximate Ramsey property and extreme amenability. For the rest of this section we fix $q, k \in \mathbb{N}$. We identify as in Subsection 3.2 the dual of $\ell_{\infty}^{d}\left(M_{q}\right)$ with $\ell_{1}^{d}\left(T_{q}\right)$. We denote by $\operatorname{Tr}$ the canonical normalized trace of $q \times q$ matrices. The isomorphism between $T_{q}$ and the dual of $M_{q}$ is induced by the pairing

$$
\langle\alpha, \beta\rangle \mapsto \operatorname{Tr}(\alpha \beta) .
$$

A linear map $\eta: M_{q} \rightarrow M_{q}$ is unital completely positive (ucp) if and only if its dual $\eta^{*}: T_{q} \rightarrow T_{q}$ is trace-preserving and completely positive. (Such maps are called quantum channels in the quantum information theory literature; see $[24, \S 4.1]$.) Thus, $\eta$ is a complete order embedding if and only if $\eta^{*}$ is a trace-preserving completely positive completely contractive quotient mapping.

Let us consider now $\ell_{\infty}^{d}\left(M_{q}\right)$. Every state $\lambda$ on $\ell_{\infty}^{d}$, which can be seen as a positive element $\left(\lambda_{1}, \ldots, \lambda_{d}\right)$ of $\ell_{1}^{d}$ of norm 1 , induces a normalized trace $\operatorname{Tr}_{\lambda}$ on $\ell_{1}^{d}\left(T_{q}\right)$ defined by

$$
\operatorname{Tr}_{\lambda}\left(a_{1}, \ldots, a_{d}\right)=\lambda_{1} \operatorname{Tr}\left(a_{1}\right)+\cdots+\lambda_{d} \operatorname{Tr}\left(a_{d}\right) .
$$

We also let $T r$ be the trace on $\ell_{1}^{d}\left(T_{q}\right)$ defined by

$$
\operatorname{Tr}\left(a_{1}, \ldots, a_{d}\right)=\operatorname{Tr}\left(a_{1}\right)+\cdots+\operatorname{Tr}\left(a_{d}\right) .
$$

Definition 4.5. Adopting the notations above, for $n, d, q \geqslant 1$ we say that a linear map $\phi: \ell_{1}^{n}\left(M_{q}\right) \rightarrow$ $\ell_{1}^{d}\left(M_{q}\right)$ is trace-preserving if, for every state $\lambda$ on $\ell_{\infty}^{d}$, and for every $a \in \ell_{1}^{n}\left(M_{q}\right), \operatorname{Tr}_{\lambda}(\phi(a))=\operatorname{Tr}(a)$.

It is easy to see that a linear map $\eta: \ell_{\infty}^{d}\left(M_{q}\right) \rightarrow \ell_{\infty}^{n}\left(M_{q}\right)$ is completely positive and unital if and only if its dual map $\eta^{*}: \ell_{1}^{n}\left(M_{q}\right) \rightarrow \ell_{1}^{d}\left(M_{q}\right)$ is completely positive and trace-preserving. Thus, $\eta$ is a complete order embedding if and only if $\eta^{*}$ is a trace-preserving completely positive complete quotient mapping. 
Let $\sigma_{d}$ be the $M_{q}$-state on $\ell_{\infty}^{d}\left(M_{q}\right)$ mapping $\left(x_{1}, \ldots, x_{d}\right)$ to $x_{d}$. A linear map $\eta: \ell_{\infty}^{d}\left(M_{q}\right) \rightarrow \ell_{\infty}^{n}\left(M_{q}\right)$ has the property that $\sigma_{n} \circ \eta=\sigma_{d}$ if and only if $\eta^{*}(0, \ldots, 0, x)=(0, \ldots, 0, x)$ for every $x \in T_{q}$. We denote by $\mathrm{TPCQ}^{M_{q}}\left(\ell_{1}^{n}\left(T_{q}\right), \ell_{1}^{d}\left(T_{q}\right)\right)$ the space of trace-preserving completely positive completely contractive quotient mappings $\phi$ from $\ell_{1}^{n}\left(T_{q}\right)$ to $\ell_{1}^{d}\left(T_{q}\right)$ such that $\phi(0, \ldots, 0, x)=(0, \ldots, 0, x)$ for every $x \in T_{q}$.

Lemma 4.6. Suppose that $\psi_{1}, \ldots, \psi_{d-1}, \phi_{d}: M_{q} \rightarrow M_{q}$ are completely positive linear maps such that $\|y-1\|<\varepsilon$, where $y=\psi_{1}(1)+\cdots+\psi_{d-1}(1)+\phi_{d}(1)$. Then there exists a completely positive map $\psi_{d}: M_{q} \rightarrow M_{q}$ such that

$$
\psi_{1}(1)+\cdots+\psi_{d-1}(1)+\psi_{d}(1)=1
$$

and $\left\|\psi_{d}-\phi_{d}\right\|<\varepsilon$.

Proof. Fix any state $s$ on $M_{q}$ and define $\psi_{d}(x)=\phi_{d}(x)+s(x)(1-y)$.

Proposition 4.7. Fix $q \in \mathbb{N}$. For any $d, m, r \in \mathbb{N}$ and $\varepsilon>0$ there exists $n \in \mathbb{N}$ such that for any $r$-coloring of $\mathrm{TPCQ}^{M_{q}}\left(\ell_{1}^{n}\left(T_{q}\right), \ell_{1}^{d}\left(T_{q}\right)\right)$ there exists $\gamma \in \operatorname{TPCQ}^{M_{q}}\left(\ell_{1}^{n}\left(T_{q}\right), \ell_{1}^{m}\left(T_{q}\right)\right)$ such that $\mathrm{TPCQ}^{M_{q}}\left(\ell_{1}^{m}\left(T_{q}\right), \ell_{1}^{d}\left(T_{q}\right)\right) \circ \gamma$ is $\varepsilon$-monochromatic.

Proof. The proof is analogous to the proof of Lemma 3.5. Fix $d, m, r \in \mathbb{N}$ and $\varepsilon>0$. We identify a linear map $\phi$ from $\ell_{1}^{n}\left(M_{q}\right)$ to $\ell_{1}^{d}\left(M_{q}\right)$ with a $d \times n$ matrix $\left[\phi_{i j}\right]$ where $\phi_{i j}: T_{q} \rightarrow T_{q}$ is a linear map. It follows from (the dual of) Lemma 3.6 that $\phi \in \mathrm{TPCQ}^{M_{q}}\left(\ell_{1}^{n}\left(T_{q}\right), \ell_{1}^{d}\left(T_{q}\right)\right)$ if and only if

- every row of $\left[\phi_{i j}\right]$ has an entry that is an automorphism of $T_{q}$,

- every column is a trace-preserving completely positive completely contractive map from $T_{q}$ to $\ell_{1}^{d}\left(T_{q}\right)$,

- the last column is $\left(0,0, \ldots, 0, \operatorname{Id}_{T_{q}}\right)$, where $\operatorname{Id}_{T_{q}}$ is the identity map of $T_{q}$.

Fix $\varepsilon_{0} \in(0, \varepsilon)$ small enough, and a finite $\varepsilon_{0}$-dense subset $U$ of the group of automorphisms of $T_{q}$ containing the identity map of $T_{q}$. The dual statement of Lemma 4.6 and the small perturbation lemma [54, Lemma 2.13.2] show that one can find a finite set $\mathcal{P}$ of trace-preserving completely positive completely contractive maps from $T_{q}$ to $\ell_{1}^{d}\left(T_{q}\right)$ with the following properties:

(1) for every $i \leqslant d$ the canonical embedding of $T_{q}$ into the $i$-th coordinate of $\ell_{1}^{d}\left(T_{q}\right)$ belongs to $\mathcal{P}$.

(2) For every trace-preserving completely positive completely contractive map $v=\left(v_{1}, \ldots, v_{d}\right): T_{q} \rightarrow$ $\ell_{1}^{d}\left(T_{q}\right)$ such that $\left(v_{1}, \ldots, v_{d-1}\right)$ is nonzero, there is a trace-preserving completely positive completely contractive map $w=\left(w_{1}, \ldots, w_{d}\right)$ in $\mathcal{P}$ such that $\|w-v\|_{\mathrm{cb}}<\varepsilon_{0},\left(w_{1}, \ldots, w_{d-1}\right)$ is nonzero, and $\left\|\left(w_{1}, \ldots, w_{d-1}\right)\right\|_{\mathrm{cb}}<\left\|\left(v_{1}, \ldots, v_{d-1}\right)\right\|_{\mathrm{cb}}$.

Let $\mathcal{Q}$ be the (finite) set of trace-preserving completely positive completely contractive quotient mappings from $\ell_{1}^{d}\left(T_{q}\right)$ to $\ell_{1}^{m}\left(T_{q}\right)$ such that the last row is $\left(0,0, \ldots, \operatorname{Id}_{T_{q}}\right)$, every column contains exactly one nonzero entry, every row contains at most one nonzero entry, and every nonzero entry is an automorphism of $T_{q}$ that belongs to $U$. Fix any linear order on $\mathcal{Q}$, and a linear order on $\mathcal{P}$ with the property that $v<w$ whenever $\left\|\left(v_{1}, \ldots, v_{d-1}\right)\right\|_{\mathrm{cb}}<\left\|\left(w_{1}, \ldots, w_{d-1}\right)\right\|_{\mathrm{cb}}$. Endow $\mathcal{Q} \times \mathcal{P}$ with the corresponding antilexicographic order. Suppose now that $n \in \mathbb{N}$ is obtained from $\mathcal{P}$ and $\mathcal{Q} \times \mathcal{P}$ by applying the dual Ramsey Theorem 2.17. We claim that $n+1$ satisfies the desired conclusions. An element of $\operatorname{Epi}(n, \mathcal{P})$ is a tuple $\bar{v}=\left(v^{(1)}, \ldots, v^{(n)}\right)$ of elements of $\mathcal{P}$. We associate with such a tuple the element $\alpha_{\bar{v}}$ of $\operatorname{TPCQ}^{M_{q}}\left(\ell_{1}^{n+1}\left(T_{q}\right), \ell_{1}^{d}\left(T_{q}\right)\right)$ whose $i$-th column is $v^{(i)}$ for $i=1,2, \ldots, n$ and the $(n+1)$-th column is $\left(0,0, \ldots, \operatorname{Id}_{T_{q}}\right)$. Similarly an element of $\operatorname{Epi}(n, \mathcal{Q} \times \mathcal{P})$ is an $n$-tuple $(\bar{B}, \bar{v})=\left(B_{1}, v_{1}, \ldots, B_{n}, v_{n}\right)$. We associate with such a tuple the completely positive completely contractive quotient mapping $\alpha_{(\bar{B}, \bar{v})}$ from $\ell_{1}^{n+1}\left(T_{q}\right)$ to $\ell_{1}^{m}\left(T_{q}\right)$ whose $i$-th column is $B_{i} v_{i}$ for $i \leqslant n$, and $\left(0,0, \ldots, 0, \operatorname{Id}_{T_{q}}\right)$ for $i=n+1$. Suppose that $c$ is an $r$-coloring of $\operatorname{TPCQ}^{M_{q}}\left(\ell_{1}^{n+1}\left(T_{q}\right), \ell_{1}^{d}\left(T_{q}\right)\right)$. 
The identification of $\operatorname{Epi}(n, \mathcal{P})$ with a subspace of $\operatorname{TPCQ}^{M_{q}}\left(\ell_{1}^{n+1}\left(T_{q}\right), \ell_{1}^{d}\left(T_{q}\right)\right)$ described above induces an $r$-coloring on $\operatorname{Epi}(n, \mathcal{P})$. By the choice of $n$ there exists an element $(\bar{B}, \bar{w})$ of $\operatorname{Epi}(n, \mathcal{Q} \times \mathcal{P})$ such that any rigid surjection from $n$ to $\mathcal{P}$ that factors through $(\bar{B}, \bar{w})$ has a fixed color $i \in r$. To conclude the proof, it remains to show that the set of elements of $\operatorname{TPCQ}^{M_{q}}\left(\ell_{1}^{n+1}\left(T_{q}\right), \ell_{1}^{d}\left(T_{q}\right)\right)$ that factor through $\alpha_{(\bar{B}, \bar{w})}$ is $\varepsilon$-monochromatic. By our choice of $n$ this will follow once we show that, given any $\rho \in \operatorname{TPCQ}^{M_{q}}\left(\ell_{1}^{m}\left(T_{q}\right), \ell_{1}^{d}\left(T_{q}\right)\right)$, there exists $\tau \in \operatorname{Epi}(\mathcal{Q} \times \mathcal{P}, \mathcal{P})$ such that $\left\|\alpha_{\tau \circ(\bar{B}, \bar{w})}-\rho \circ \alpha_{(\bar{B}, \bar{w})}\right\|_{\mathrm{cb}} \leqslant \varepsilon$. Here we denoted by $\tau \circ(\bar{B}, \bar{w})$ the rigid surjection from $n$ to $\mathcal{P}$ that one obtains by composing $(\bar{B}, \bar{w})-$ regarded as a rigid surjection - and $\tau$. If $\rho$ has representative matrix $A$, this is equivalent to the assertion that for every $i \leqslant n,\left\|A B_{i} w_{i}-\tau\left(B_{i}, w_{i}\right)\right\|_{\mathrm{cb}} \leqslant \varepsilon$. We proceed to define such a rigid surjection $\tau$ from $\mathcal{Q} \times \mathcal{P}$ to $\mathcal{P}$. By the structure of completely positive completely contractive quotient mappings from $\ell_{1}^{m}\left(T_{q}\right)$ to $\ell_{1}^{d}\left(T_{q}\right)$ recalled above, there exists $A^{\dagger} \in \mathcal{Q}$ such that $\left\|A A^{\dagger}-\operatorname{Id}_{\ell^{1}}\left(T_{q}\right)\right\|_{\mathrm{cb}} \leqslant \varepsilon$, provided that $\varepsilon_{0}$ is small enough. Define now $\tau: \mathcal{Q} \times \mathcal{P} \rightarrow \mathcal{P}$ by letting, for $B \in \mathcal{Q}$ and $w=\left(w_{1}, \ldots, w_{d}\right) \in \mathcal{P}$, if $A B w=v=\left(v_{1}, \ldots, v_{d}\right), \tau(B, w):=\widetilde{v}=\left(\widetilde{v}_{1}, \ldots, \widetilde{v}_{d}\right)$ such that:

- if $B=A^{\dagger}$ or if $v=\left(0,0, \ldots, 0, v_{d}\right)$, then $\widetilde{v}=w$;

- otherwise, $\widetilde{v}$ is an element of $\mathcal{P}$ such that $\|\widetilde{v}-v\|_{\mathrm{cb}} \leqslant \varepsilon,\left(\widetilde{v}_{1}, \ldots, \widetilde{v}_{d-1}\right)$ is nonzero, and we have that $\left\|\left(\widetilde{v}_{1}, \ldots, \widetilde{v}_{d-1}\right)\right\|_{\mathrm{cb}}<\left\|\left(v_{1}, \ldots, v_{d-1}\right)\right\|_{\mathrm{cb}}$.

It is clear from the definition that $\|\tau(B, w)-A B w\|_{\mathrm{cb}} \leqslant \varepsilon$ for every $(B, w) \in \mathcal{Q} \times \mathcal{P}$. We need to verify that $\tau$ is indeed a rigid surjection from $\mathcal{Q} \times \mathcal{P}$ to $\mathcal{P}$. Observe that $\tau$ is onto. Fix $\widetilde{v}=\left(\widetilde{v}_{1}, \ldots, \widetilde{v}_{d}\right) \in$ $\mathcal{P}$. If $\widetilde{v}=\left(0, \ldots, 0, \widetilde{v}_{d}\right)$, then the least element of $\mathcal{Q} \times \mathcal{P}$ that is mapped by $\tau$ to $\widetilde{v}$ is $(B, \widetilde{v})$, where $B$ is the least element of $\mathcal{Q}$. If $\left(\widetilde{v}_{1}, \ldots, \widetilde{v}_{d-1}\right)$ is not the zero vector, then the least element of $\mathcal{Q} \times \mathcal{P}$ that is mapped by $\tau$ to $\widetilde{v}$ is $\left(A^{\dagger}, \widetilde{v}\right)$. Indeed, suppose that $(B, w)$ is an element of the preimage of $\widetilde{v}$ under $\tau$ such that $B$ is different from $A^{\dagger}$. Set $A B w=v=\left(v_{1}, \ldots, v_{d}\right)$. By definition of $\tau$ we have that

$$
\left\|\left(\widetilde{v}_{1}, \ldots, \widetilde{v}_{d-1}\right)\right\|_{\mathrm{cb}}<\left\|\left(v_{1}, \ldots, v_{d-1}\right)\right\|_{\mathrm{cb}} \leqslant\left\|\left(w_{1}, \ldots, w_{d-1}\right)\right\|_{\mathrm{cb}} .
$$

Therefore by definition of the order on $\mathcal{P}$ and on $\mathcal{Q} \times \mathcal{P}$ we have that $\widetilde{v}<w$ and $\left(A^{\dagger}, \widetilde{w}\right)<(B, w)$. This concludes the proof that the least element of $\mathcal{Q} \times \mathcal{P}$ that is mapped by $\tau$ to $\widetilde{v}$ is $\left(A^{\dagger}, \widetilde{v}\right)$. These remarks clearly imply that $\tau$ is a rigid surjection.

The following result can be proved from Proposition 4.7 similarly as Theorem 3.13.

Theorem 4.8. The following classes of finite-dimensional R-operator systems satisfy the stable Ramsey property with modulus $\varpi(\delta)=3 \delta$ :

(1) for every $q \in \mathbb{N}$ the class $\left\{\left(\ell_{\infty}^{d}\left(M_{q}\right), s_{d}\right)\right\}_{d \in \mathbb{N}}$ of $M_{q}$-operator systems, where $s_{d}\left(x_{1}, \ldots, x_{d}\right)=x_{d}$.

(2) For every $q \in \mathbb{N}$ and $q$-minimal separable nuclear operator system $R$, the class of finite-dimensional q-minimal $R$-operator systems.

(3) For every separable nuclear operator system $R$, the class of finite-dimensional exact $R$-operator systems.

The limits of the Fraïssé classes mentioned in Theorem 4.8 have extremely amenable automorphism groups in view of the correspondence between extreme amenability and the approximate Ramsey property given by Proposition 2.16.

Corollary 4.9. The following Polish groups are extremely amenable:

(1) the stabilizer $\operatorname{Aut}\left(A(\mathbb{P}), \Omega_{A(\mathbb{P})}^{A(F)}\right)$ of the generic $A(F)$-state $\Omega_{A(\mathbb{P})}^{A(F)}$ on the Poulsen system $A(\mathbb{P})$ for any metrizable Choquet simplex $F$ [3, Theorem 3.3]. 
(2) The stabilizer $\operatorname{Aut}\left(A(\mathbb{N P}), \Omega_{A(\mathbb{N P})}^{R}\right)$ of the generic R-state $\Omega_{A(\mathbb{N P})}^{R}$ on the noncommutative Poulsen system $A(\mathbb{N P})$ for any separable nuclear operator system $R$.

(3) The stabilizer $\operatorname{Aut}\left(A\left(\mathbb{P}^{(q)}\right), \Omega_{A\left(\mathbb{P}^{(q)}\right)}^{R}\right)$ of the generic R-state $\Omega_{A\left(\mathbb{P}^{(q)}\right)}^{R}$ on the q-minimal Poulsen system $A\left(\mathbb{P}^{(q)}\right)$ for any q-minimal nuclear operator system $R$.

4.3. The universal minimal flows of the Aut $(\mathbb{N P})$. Using Corollary 4.9 we can compute the universal minimal flows of the matrix affine homeomorphism group Aut $(\mathbb{N P})$ of the noncommutative Poulsen simplex, and the matrix affine homeomorphism group $\operatorname{Aut}\left(\mathbb{P}^{(q)}\right)$ of the $q$-minimal Poulsen simplex. The corresponding result for the Poulsen simplex was obtained in [3, Theorem 3.10].

\section{Theorem 4.10.}

(1) The universal minimal flow of $\operatorname{Aut}(\mathbb{N P})$ is the canonical action $\operatorname{Aut}(\mathbb{N P}) \curvearrowright \mathbb{N P}_{1}$.

(2) The universal minimal flow of $\operatorname{Aut}\left(\mathbb{P}^{(q)}\right)$ is the canonical action $\operatorname{Aut}\left(\mathbb{P}^{(q)}\right) \curvearrowright \mathbb{P}_{1}^{(q)}$.

Proof. (1): The minimality of the action $\operatorname{Aut}(\mathbb{N P}) \curvearrowright \mathbb{N P}_{1}$ is a consequence of the following fact: for any $d \in \mathbb{N}$ and $\varepsilon>0$ there exists $m \in \mathbb{N}$ such that for any $s \in S\left(M_{d}\right)$ and $t \in S\left(M_{m}\right)$ there exists a complete order embedding $\phi: M_{d} \rightarrow M_{m}$ such that $\|t \circ \phi-s\|_{\mathrm{cb}}<\varepsilon$; see [37, Lemma 8.10] and [37, Proposition 5.8]. Consider the generic state $\Omega_{A(\mathbb{N} P)}^{\mathbb{C}}$ on $A(\mathbb{N P})$. It is shown in $\left[37\right.$, Section 8.2] that $\Omega_{A(\mathbb{N P})}^{\mathbb{C}}$ is a matrix extreme point of $\mathbb{N P}$ whose Aut $(\mathbb{N P})$-orbit is dense in $\mathbb{N P}_{1}$. The stabilizer Aut $\left(\mathbb{N P}, \Omega_{A(\mathbb{N} P)}^{\mathbb{C}}\right)$ of $\Omega_{A(\mathbb{N P})}^{\mathbb{C}}$ is extremely amenable by Corollary 4.9. The canonical Aut $(\mathbb{N P})$-equivariant map from the quotient $\operatorname{Aut}(\mathbb{N P})$-space $\operatorname{Aut}(\mathbb{N P}) / / \operatorname{Aut}\left(\mathbb{N P}, \Omega_{A(\mathbb{N} P)}^{\mathbb{C}}\right)$ to $\mathbb{N P}_{1}$ is a uniform equivalence. This follows from the homogeneity property of $\left(A(\mathbb{N P}), \Omega_{A(\mathbb{N} P)}^{\mathbb{C}}\right)$ as the Fraïssé limit of the class of finite-dimensional operator systems with a distinguished state; see also [37, Subsection 5.4]. This allows one to conclude via a standard argument - see $[43$, Theorem1.2] - that the action $\operatorname{Aut}(\mathbb{N P}) \curvearrowright \mathbb{N P}$ is the universal minimal compact Aut $(\mathbb{N P})$-space.

(2): Minimality of the action $\operatorname{Aut}\left(\mathbb{P}^{(q)}\right) \curvearrowright \mathbb{P}_{1}^{(q)}$ is a consequence of a similar assertion than in 1), where $M_{d}$ and $M_{m}$ are replaced with $\ell_{\infty}^{d}\left(M_{q}\right)$ and $\ell_{\infty}^{m}\left(M_{q}\right)$; see [37, Lemma 6.25]. The rest of the argument is entirely analogous.

\section{REFERENCES}

[1] Erik M. Alfsen. Compact convex sets and boundary integrals. Springer-Verlag, New YorkHeidelberg, 1971.

[2] Dana Bartošová, Jordi Lopez-Abad, Martino Lupini, and Brice Mbombo. The Ramsey properties for Grassmannians over $\mathbb{R}, \mathbb{C}$. Preprint arXiv:1910.00311, 2019.

[3] Dana Bartošová, Jordi Lopez-Abad, Martino Lupini, and Brice Mbombo. The Ramsey property for Banach spaces and Choquet simplices. Journal of the European Mathematical Society, 2019. to appear; arXiv:1708.01317.

[4] Itaï Ben Yaacov. Fraïssé limits of metric structures. Journal of Symbolic Logic, 80(1):100-115, 2015.

[5] Itaï Ben Yaacov, Alexander Berenstein, C. Ward Henson, and Alexander Usvyatsov. Model theory for metric structures. In Model theory with applications to algebra and analysis. Vol. 2, volume 350 of London Mathematical Society Lecture Note Series, pages 315-427. Cambridge University Press, 2008. 
[6] David P. Blecher and Christian Le Merdy. Operator algebras and their modules - an operator space approach, volume 30 of London Mathematical Society Monographs. New Series. Oxford University Press, Oxford, 2004.

[7] David P. Blecher and Matthew Neal. Metric characterizations of isometries and of unital operator spaces and systems. Proceedings of the American Mathematical Society, 139(3):985-998, 2011.

[8] Man-Duen Choi and Edward G. Effros. Injectivity and operator spaces. Journal of Functional Analysis, 24(2):156-209, 1977.

[9] Kenneth R. Davidson and Matthew Kennedy. Noncommutative Choquet theory. arXiv:1905.08436, December 2019. arXiv: 1905.08436.

[10] Caleb Eckhardt. Perturbations of completely positive maps and strong NF algebras. Proceedings of the London Mathematical Society, 101(3):795-820, 2010.

[11] Edward G. Effros. Aspects of noncommutative order. In $C^{*}$-algebras and applications to physics (Proc. Second Japan-USA Sem., Los Angeles, Calif., 1977), volume 650 of Lecture Notes in Mathematics, pages 1-40. Springer, Berlin, 1978.

[12] Edward G. Effros. A matrix convexity approach to some celebrated quantum inequalities. Proceedings of the National Academy of Sciences of the United States of America, 106(4):1006-1008, 2009.

[13] Edward G. Effros and Zhong-Jin Ruan. Operator spaces, volume 23 of London Mathematical Society Monographs. New Series. Oxford University Press, 2000.

[14] Edward G. Effros and Soren Winkler. Matrix convexity: operator analogues of the bipolar and Hahn-Banach theorems. Journal of Functional Analysis, 144(1):117-152, February 1997.

[15] Robert Ellis. Universal minimal sets. Proceedings of the American Mathematical Society, 11:540543, 1960.

[16] Douglas R. Farenick. Extremal matrix states on operator systems. Journal of the London Mathematical Society, 61(3):885-892, 2000.

[17] Douglas R. Farenick. Pure matrix states on operator systems. Linear Algebra and its Applications, 393:149-173, 2004.

[18] Valentin Ferenczi, Jordi Lopez-Abad, Brice Mbombo, and Stevo Todorcevic. Amalgamation and Ramsey properties of $L_{p}$ spaces. Adv. Math., 369:107190, 76, 2020.

[19] Adam H. Fuller, Michael Hartz, and Martino Lupini. Boundary representations of operator spaces and compact rectangular matrix convex sets. Journal of Operator Theory, 79(1):139-172, 2018.

[20] Isaac Goldbring and Martino Lupini. Model-theoretic aspects of the Gurarij operator space. Israel Journal of Mathematics. in press.

[21] Isaac Goldbring and Thomas Sinclair. On Kirchberg's embedding problem. Journal of Functional Analysis, 269(1):155-198, July 2015.

[22] Ronald L. Graham and Bruce L. Rothschild. Ramsey's theorem for $n$-parameter sets. Transactions of the American Mathematical Society, 159, 1971.

[23] Mikhael Gromov and Vitali D. Milman. A topological application of the isoperimetric inequality. American Journal of Mathematics, 105(4):843-854, 1983.

[24] Ved Prakash Gupta, Prabha Mandayam, and V. S. Sunder. The functional analysis of quantum information theory, volume 902 of Lecture Notes in Physics. Springer, Cham, 2015.

[25] Vladimir I. Gurariı. Spaces of universal placement, isotropic spaces and a problem of Mazur on rotations of Banach spaces. Siberian Mathematical Journal, 7:1002-1013, 1966. 
[26] Yonatan Gutman and Hanfeng Li. A new short proof for the uniqueness of the universal minimal space. Proceedings of the American Mathematical Society, 141(1):265-267, 2013.

[27] J. William Helton, Igor Klep, and Scott McCullough. The tracial Hahn-Banach theorem, polar duals, matrix convex sets, and projections of free spectrahedra. Journal of the European Mathematical Society, 19(6):1845-1897, 2017.

[28] William Helton, Igor Klep, and Scott McCullough. Free convex algebraic geometry. In Semidefinite optimization and convex algebraic geometry, volume 13 of MOS-SIAM Ser. Optim., pages 341-405. SIAM, Philadelphia, PA, 2013.

[29] Ali S. Kavruk, Vern I. Paulsen, Ivan G. Todorov, and Mark Tomforde. Quotients, exactness, and nuclearity in the operator system category. Advances in Mathematics, 235:321-360, 2013.

[30] Alexander S. Kechris, Vladimir Pestov, and Stevo Todorcevic. Fraïssé limits, Ramsey theory, and topological dynamics of automorphism groups. Geometric and Functional Analysis, 15(1):106189, 2005.

[31] Eberhard Kirchberg and Simon Wassermann. C*-algebras generated by operator systems. Journal of Functional Analysis, 155(2):324-351, 1998.

[32] Wiesław Kubiś and Sławomir Solecki. A proof of uniqueness of the Gurariŭ space. Israel Journal of Mathematics, 195(1):449-456, 2013.

[33] Anselm Lambert. Operatorfolgenräume. PhD thesis, Universität des Saarlandes, 2002.

[34] Franz Lehner. $M_{n}$-espaces, sommes d'unitaires et analyse harmonique sur le groupe libre. PhD thesis, Université de Paris 6, 1997.

[35] Martino Lupini. Operator space and operator system analogs of Kirchberg's nuclear embedding theorem. Journal of Mathematical Analysis and Applications, 431(1):47-56, 2015.

[36] Martino Lupini. Uniqueness, universality, and homogeneity of the noncommutative Gurarij space. Advances in Mathematics, 298:286-324, August 2016.

[37] Martino Lupini. Fraïssé limits in functional analysis. Advances in Mathematics, 338:93-174, 2018.

[38] Martino Lupini. The Kirchberg-Wassermann operator system is unique. Journal of Mathematical Analysis and Applications, 459(2):1251-1259, 2018.

[39] Wolfgang Lusky. The Gurarij spaces are unique. Archiv der Mathematik, 27(6):627-635, 1976.

[40] Wolfgang Lusky. On separable Lindenstrauss spaces. Journal of Functional Analysis, 26(2):103120, October 1977.

[41] Wolfgang Lusky. Some consequences of W. Rudin's paper: " $L_{p}$-isometries and equimeasurability". Indiana University Mathematics Journal, 27(5):859-866, 1978.

[42] Wolfgang Lusky. On a construction of Lindenstrauss and Wulbert. Journal of Functional Analysis, 31(1):42-51, January 1979.

[43] Julien Melleray, Lionel Nguyen Van Thé, and Todor Tsankov. Polish groups with metrizable universal minimal flows. International Mathematics Research Notices., (5):1285-1307, 2016.

[44] Julien Melleray and Todor Tsankov. Extremely amenable groups via continuous logic. arXiv:1404.4590, 2014.

[45] Lionel Nguyen Van Thé. More on the Kechris-Pestov-Todorcevic correspondence: precompact expansions. Fundamenta Mathematicae, 222(1):19-47, 2013.

[46] Timur Oikhberg. The non-commutative Gurarii space. Archiv der Mathematik, 86(4):356-364, 2006. 
[47] Carlos Palazuelos and Thomas Vidick. Survey on Nonlocal Games and Operator Space Theory. Journal of Mathematical Physics, 57(1):015220, January 2016.

[48] Vern I. Paulsen. Completely bounded maps and operator algebras, volume 78 of Cambridge Studies in Advanced Mathematics. Cambridge University Press, Cambridge, 2002.

[49] Vladimir Pestov. The isometry group of the Urysohn space as a Lévy group. 154(10):2173-2184.

[50] Vladimir Pestov. Ramsey-Milman phenomenon, Urysohn metric spaces, and extremely amenable groups. Israel Journal of Mathematics, 127(1):317-357, 2002.

[51] Vladimir Pestov. Dynamics of Infinite-dimensional Groups, volume 40 of University Lecture Series. American Mathematical Society, Providence, RI, 2006.

[52] Gilles Pisier. Exact operator spaces. Astérisque, (232):159-186, 1995. Recent advances in operator algebras (Orléans, 1992).

[53] Gilles Pisier. The operator Hilbert space OH, complex interpolation and tensor norms. Memoirs of the American Mathematical Society, 122(585):viii+103, 1996.

[54] Gilles Pisier. Introduction to operator space theory, volume 294 of London Mathematical Society Lecture Note Series. Cambridge University Press, Cambridge, 2003.

[55] Ebbe T. Poulsen. A simplex with dense extreme points. Annales de l'Institut Fourier, 11:83-87, 1961.

[56] Gideon Schechtman. Almost isometric $L_{p}$ subspaces of $L_{p}(0,1)$. The Journal of the London Mathematical Society, 20(3):516-528, 1979.

[57] Roger R. Smith. Finite dimensional injective operator spaces. Proceedings of the American Mathematical Society, 128(11):3461-3462, 2000.

[58] Corran Webster and Soren Winkler. The Krein-Milman theorem in operator convexity. Transactions of the American Mathematical Society, 351(1):307-322, 1999.

[59] Przemysław Wojtaszczyk. Some remarks on the Gurarij space. Studia Mathematica, 41:207-210, 1972.

[60] Blerina Xhabli. The super operator system structures and their applications in quantum entanglement theory. Journal of Functional Analysis, 262(4):1466-1497, 2012.

Department of Mathematics, University of Florida, PO Box 118105, Gainesville, FL 32611.

Email address: dbartoso@andrew.cmu.edu

Departamento de Matemáticas Fundamentales, Facultad de Ciencias, Uned, 28040 Madrid, Spain

Email address: abad@mat.uned.es

School of Mathematics and Statistics, Victoria University of Wellington, PO Box 600, Wellington 6140, NEW ZEALAND

Email address: martino.lupini@vuw.ac.nz

$U R L:$ http://www.lupini.org/

Collège Lionel-Groulx, 100 rue Duquet, Sainte-Therese, QC J7E3G6, Canada

Email address: BR.MDempowo@glc.qc.ca

Departamento de Matemática, Instituto de Matemática e Estatí́stica, Universidade de São Paulo, rua Do Matão, 1010, 05508-090 SÃo PAUlo, SP, BRAZIL 